Bull. Korean Math. Soc. 49 (2012), No. 5, pp. 1101-1129

http://dx.doi.org/10.4134/BKMS.2012.49.5.1101

\title{
HOMOGENEOUS SUBMERSIONS OF 3-DIMENSIONAL GEOMETRIES
}

\author{
Kyung Bai Lee and Joonsang Park
}

\begin{abstract}
We study the geometry of the images of 1-dimensional homogeneous submersions for each of the model spaces $X$ of the eight 3dimensional geometries. In particular, We shall calculate the group of isometries and the curvatures of the base surfaces for each of the model spaces of 3-dimensional geometries, with respect to every closed subgroup of the isometries of $X$ acting freely.
\end{abstract}

\section{Introduction}

The goal of this paper is to understand the geometry of the images of 1dimensional homogeneous submersions for each of the model spaces of the eight 3 -dimensional geometries [5]. More precisely, let $X$ be one of the model spaces, and $G$ be a closed subgroup of $\operatorname{Isom}(X)$ acting freely on $X$. Note that a free action of a 1-dimensional closed subgroup $G$ of the isometry group of Riemannian manifold $X$ is automatically proper. Thus, the action gives rise to a homogeneous foliation. Let $G \backslash X$ denote the orbit space. We have a Riemannian submersion (principal $G$-bundle)

$$
G \longrightarrow X \stackrel{\pi}{\longrightarrow} G \backslash X \text {. }
$$

$G$ can be interpreted as a group as well as a homogeneous foliation.

The goal of this paper is to study the spaces $G \backslash X$. In particular, we shall calculate the group of isometries and the curvatures of $G \backslash X$, for each of the model spaces of 3-dimensional geometries, with respect to every closed subgroup of $\operatorname{Isom}(X)$ acting freely on $X$.

Suppose $G$ and $G^{\prime}$ are two subgroups of $\operatorname{Isom}(X)$ such that $G^{\prime}=f G f^{-1}$ for some $f \in \operatorname{Isom}(X)$. Then the map $f$ induces an isometry from $G \backslash X$ to $G^{\prime} \backslash X$. Therefore, we need to classify the conjugacy classes of all 1-dimensional closed

Received August 16, 2011.

2010 Mathematics Subject Classification. 53C 30, 53C12.

Key words and phrases. homogeneous, submersion, orbit space, foliation, 8-geometry, Gaussian curvature.

The second author was supported by Basic Science Research Program through the National Research Foundation of Korea(NRF) funded by the Ministry of Education, Science and Technology 2010-0009632. 
subgroups of $\operatorname{Isom}_{0}(X)$ that can act freely. Then for each such $G$, we parametrize it, and find the global vertical vector field $V$. Since different parametrizations give the same results, we will work with subgroups, up to conjugacy and up to parametrization. Next we find an orthonormal frame $\left\{V /\|V\|, X_{1}, X_{2}\right\}$. The distribution $\left\{X_{1}, X_{2}\right\}$ will be mapped to $G \backslash X$ isometrically by the projection map $\pi$. In general, it is hard to write out the projection map $\pi$ explicitly. However, we can still find $\left\{\pi_{*}\left(X_{1}\right), \pi_{*}\left(X_{2}\right)\right\}$, an orthonormal basis for $G \backslash X$. This gives the Riemannian metric on $G \backslash X$.

Once the Riemannian metric of $G \backslash X$ is found, the group of isometries and the curvature of $G \backslash X$ can be calculated. However, for these two items, one really does not need to know the metric on $G \backslash X$ explicitly. By calculating the normalizer of the subgroup $G$ in $\operatorname{Isom}(X)$, we can find $\operatorname{Isom}(G \backslash X)$. The curvature $\kappa\left(\pi_{*} X_{1}, \pi_{*} X_{2}\right)$ on $G \backslash X$ can also be calculated by O'Neill formula.

0.1. Since the explicit calculation of $\pi_{*}$ is important, we shall describe it here in detail. We assume $X$ is not $S^{3}$ ( $S^{3}$ will be treated differently). Suppose

$$
\varphi: \mathbb{R}^{3} \longrightarrow X
$$

is a local trivialization such that $\varphi(\bullet, \bullet, 0)$ yields a local cross section for the Riemannian submersion $\pi: X \longrightarrow B$. We denote the image of this cross section by $\hat{B}$, i.e.,

$$
\hat{B}=\left\{\varphi(x, y, 0):(x, y, 0) \in \mathbb{R}^{3}\right\} \subset X
$$

(again this may not be global). Define a map $\psi: X \longrightarrow X$ by

$$
\psi(\varphi(x, y, t))=\xi(t) \cdot \varphi(x, y, 0) .
$$

If we set $\hat{\psi}=\varphi^{-1} \circ \psi \circ \varphi: \mathbb{R}^{3} \longrightarrow \mathbb{R}^{3}$, then the following diagram

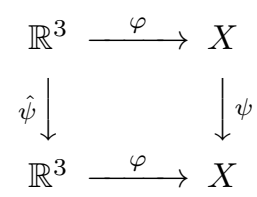

is commutative.

0.2. Clearly, $\psi$ leaves the subset $\hat{B}$ pointwise fixed. At any $p=\varphi(x, y, 0) \in \hat{B}$, $\xi(t) \cdot p$ is the leaf passing through $p$. Then

$$
V=\left.\frac{d}{d t}(\xi(t) \cdot p)\right|_{t=0}
$$

is the vertical vector of the Riemannian projection. We can find tangent vectors $X_{1}, X_{2} \in T_{p}(X)$ so that

$$
\left\{V /\|V\|, X_{1}, X_{2}\right\}
$$

forms an orthonormal basis of $T_{p}(X)$. Then $\left\{\pi_{*}\left(X_{1}\right), \pi_{*}\left(X_{2}\right)\right\}$ is an orthonormal basis of $T_{\pi_{*}}(p)$. 
0.3 (Jacobian of the projection). By defining a new Riemannian metric on $\hat{B}$ (not as a subspace of $X$ ), we shall identify $\hat{B}$ with $B$, and make

$$
\xi(t) \cdot \varphi(x, y, 0) \mapsto \varphi(x, y, 0)
$$

a Riemannian submersion. To this end, it is enough to declare that the set of the two tangent vectors

$$
\left\{\psi_{*}\left(X_{1}\right), \psi_{*}\left(X_{2}\right)\right\} \subset T_{p}(\hat{B})
$$

is an orthonormal basis. On $\mathbb{R}^{3}$, this is the Jacobian matrix

$$
J=\left.\left(\begin{array}{lll}
1 & 0 & 0 \\
0 & 1 & 0
\end{array}\right)\left(\left[\frac{\partial \varphi^{-1}(\xi(t) \cdot \varphi(x, y, 0))}{\partial(x, y, t)}\right]^{-1}\right)^{t}\right|_{t=0} .
$$

0.4 (Curvature). Once we find an orthonormal basis, we understand the quotient space. In particular, calculating the curvature is routine: Let $\omega_{1}, \omega_{2}$ be the coframe field dual to $J\left(X_{1}\right), J\left(X_{2}\right)$. The connection 1-form $\omega_{12}=-\omega_{21}$ is defined by

$$
\begin{aligned}
& d \omega_{1}=\omega_{12} \wedge \omega_{2}, \\
& d \omega_{2}=\omega_{21} \wedge \omega_{1} .
\end{aligned}
$$

Then the curvature $\kappa$ is obtained from

$$
d \omega_{12}=-\kappa \omega_{1} \wedge \omega_{2} .
$$

The curvature $\kappa\left(\pi_{*} X_{1}, \pi_{*} X_{2}\right)$ on $G \backslash X$ can be calculated also by O'Neill formula [3].

$$
\kappa\left(\pi_{*} X_{1}, \pi_{*} X_{2}\right)=\tilde{\kappa}\left(X_{1}, X_{2}\right)+3\left\|A_{X_{1}} X_{2}\right\|^{2},
$$

where $\tilde{\kappa}$ is the sectional curvature of the total space $X$, and $A_{X_{1}} X_{2}=\frac{1}{2}\left[X_{1}, X_{2}\right]^{v}$ is the vertical component of $\frac{1}{2}\left[X_{1}, X_{2}\right]$. Note that this method does not require the Jacobian.

We made calculation of curvatures in two ways, first directly after finding $\left\{J\left(X_{1}\right), J\left(X_{2}\right)\right\}$; and secondly using O'Neill Formula (0.2) using $\left\{X_{1}, X_{2}\right\}$ (not using $\left.\left\{J\left(X_{1}\right), J\left(X_{2}\right)\right\}\right)$. All calculations are done by the program Mathematica, and hand checked.

$\mathbf{0 . 5}$ (Isometry group of $G \backslash X)$. Let $N$ be the normalizer of the subgroup $G$ in Isom $(X)$, and let $N_{0}$ be the connected component of $N$ containing the identity. Since the action of $N$ on $X$ is fiber-preserving, it induces an action on the quotient $G \backslash X$. Furthermore, since $N \subset \operatorname{Isom}(X)$ and $\pi$ is a Riemannian submersion, the action of $N$ on $G \backslash X$ is as isometries. Of course, $G$ itself acts ineffectively. Thus, $N_{0} / G \subset \operatorname{Isom}_{0}(G \backslash X)$.

The group $\operatorname{Isom}_{0}(G \backslash X)$ can be determined by the above inequality together with the curvature results: $\operatorname{Isom}_{0}(G \backslash X)$ is 2-dimensional if and only if $\kappa$ is constant; $\operatorname{Isom}_{0}(G \backslash X)$ is 1-dimensional if and only if $\kappa$ depends only on one variable and not constant. To show that $\operatorname{Isom}_{0}(G \backslash X)$ is 0-dimensional, 
one needs some more argument. See Theorem 7.3. We found, in all cases, $\operatorname{Isom}_{0}(G \backslash X)=N_{0} / G$.

The twisted geometries Nil and $\widetilde{\mathrm{PSL}}_{2} \mathbb{R}$ offer the most interesting quotients. For example, in the case of $\widetilde{\mathrm{PSL}}_{2} \mathbb{R}$-geometry, the base manifold can be flat $\mathbb{R}^{2}$, hyperbolic $\mathbb{H}^{2}$, or quite complicated Riemannian manifold with bounded sectional curvature. In the first two cases, $N_{0} / G$ is 2-dimensional.

0.6 (Notation). Since the model spaces $X$ are all simply connected, $G$ must be $\mathbb{R}$ except for $S^{3}$. One dimensional Riemannian foliations on $S^{3}$ is well known, and we will assume $X$ is not $S^{3}$. The following notation will be used throughout:

$$
\begin{array}{ll}
\varphi: \mathbb{R}^{3} \rightarrow X & \text { global (local) trivialization map, } \\
\xi: \mathbb{R} \rightarrow G \subset \operatorname{Isom}_{0}(X) & \text { parametrization of the subgroup } G .
\end{array}
$$

The rotations $R(t) \in \mathrm{SO}(2), \widehat{R}(t) \in \mathrm{SO}(3)$ are

$$
R(t)=\left[\begin{array}{rr}
\cos t & -\sin t \\
\sin t & \cos t
\end{array}\right], \quad \widehat{R}(t)=\left[\begin{array}{ccc}
\cos t & -\sin t & 0 \\
\sin t & \cos t & 0 \\
0 & 0 & 1
\end{array}\right] .
$$

For a matrix $A, A^{t}$ denotes its transpose. $I_{2}$ and $I_{3}$ denote the identity matrices of size 2 and 3 , respectively.

\section{The Euclidean space $\mathbb{R}^{3}$}

The group of isometries is $\operatorname{Isom}_{0}\left(\mathbb{R}^{3}\right)=\mathbb{R}^{3} \rtimes \mathrm{SO}(3)$. The classification of 1-dimensional subgroups is easy.

Proposition 1.1. A 1-dimensional closed subgroup $G$ of $\mathbb{R}^{3} \rtimes \mathrm{SO}(3)$ which can act freely on $\mathbb{R}^{3}$ is either $\xi(t)=\left(t_{\mathbf{e}_{3}}, I_{3}\right)$ or $\xi(t)=\left(\right.$ tce $\left.\mathbf{e}_{3}, \widehat{R}(t)\right)$ with $c \neq 0$, where $\mathbf{e}_{3}=(0,0,1)^{t}$, up to conjugation.

Proof. Suppose $G$ is given by a homomorphism $\xi: \mathbb{R} \rightarrow \mathbb{R}^{3} \rtimes \mathrm{SO}(3)$. It induces a homomorphism $\mathbb{R} \rightarrow \mathrm{SO}(3)$ whose image is abelian. Therefore, the image lies in a maximal torus which is $\mathrm{SO}(2)$. Thus, without loss of generality, we may assume $\xi$ is of the form

$$
\xi(t)=(\lambda(t), \widehat{R}(\alpha t)),
$$

where $\lambda: \mathbb{R} \rightarrow \mathbb{R}^{3}$ satisfies the cocycle condition

$$
\lambda(s+t)=\lambda(s)+\widehat{R}(\alpha s)(\lambda(t)) .
$$

If $\alpha=0$, then $\lambda$ is a homomorphism. By conjugation by an element of $\operatorname{SO}(3)$, we can put $\xi(t)$ into the form $\left(t \mathbf{e}_{3}, I_{3}\right)$. If $\alpha \neq 0$, since we are interested in the group itself, not the parametrizations, we may assume $\alpha=1$ (by reparametrizing). 
Suppose $\xi\left(t_{0}\right)=\left(\lambda\left(t_{0}\right), \widehat{R}\left(t_{0}\right)\right)=(\mathbf{a}, U) \in \mathbb{R}^{3} \rtimes \mathrm{SO}(3)$, and $U \neq I_{3}$. Such $\xi$ is completely determined by $\xi\left(t_{0}\right)$. For $\mathbf{x} \in \mathbb{R}^{3}$,

$$
(\mathbf{x}, I)(\mathbf{a}, U)(\mathbf{x}, I)^{-1}=(\mathbf{x}+\mathbf{a}-U(\mathbf{x}), U) .
$$

If $\mathbf{a}=(u, v, w)^{t}$,

$$
\mathbf{x}=\left(\frac{1}{2}\left(-u+v \cot \frac{t_{0}}{2}\right), \frac{1}{2}\left(-u \cot \frac{t_{0}}{2}-v\right), 0\right)^{t}
$$

will make

$$
\mathbf{x}+\mathbf{a}-U(\mathbf{x})=(0,0, c)^{t} .
$$

Thus, conjugation of $\left(\lambda\left(t_{0}\right), \widehat{R}\left(t_{0}\right)\right)$ by our $(\mathbf{x}, I)$ is of the form $\left(t_{0} \mathbf{c e}_{3}, \widehat{R}\left(t_{0}\right)\right) \in$ $\mathbb{R}^{3} \times \mathrm{SO}(3)$. Note that $\mathrm{SO}(2)$ acts on this $\mathbb{R} \mathbf{e}_{3}$ trivially, and the semi-direct product becomes product. Consequently, we have $\xi(t)=\left(t c \mathbf{e}_{3}, \widehat{R}(t)\right)$. If $c=0$, then $\xi(t)$ has fixed points, and the action is not free.

1.2. With $\xi(t)=((0,0, c t), \widehat{R}(t))$, the leaves have the equation

$$
\xi(t) \cdot(x, y, 0)=(x \cos t-y \sin t, y \cos t+x \sin t, c t) .
$$

For the action of $\xi$ to be free, we must have $c \neq 0$. When the group $\{\xi(t)\}$ acts properly and freely, every orbit meets $\mathbb{R}^{2} \times\{0\}$ once and exactly once. Hence we can take the $x y$-plane as a global cross section.

The vertical vector $V_{(x, y, 0)}$ at $(x, y, 0) \in \mathbb{R}^{3}$ is

$$
V_{(x, y, 0)}=\left.\frac{d}{d t}\right|_{t=0} \xi(t) \cdot(x, y, 0)=(-y, x, c) .
$$

Let

$$
\begin{aligned}
& X_{1}=\frac{1}{\sqrt{c^{2}+y^{2}}}(c, 0, y), \\
& X_{2}=\frac{1}{\sqrt{\left(c^{2}+y^{2}\right)\left(c^{2}+x^{2}+y^{2}\right)}}\left(x y, c^{2}+y^{2},-c x\right) .
\end{aligned}
$$

Then $\left\{X_{1}, X_{2}\right\}$ is an orthonormal basis for the horizontal distribution at $(x, y, 0)$.

The equation of leaves (1.1) yields the Jacobian of the Riemannian projection on the $x y$-plane (as explained in subsection (0.3))

$$
J=\left[\begin{array}{rrr}
1 & 0 & \frac{y}{c} \\
0 & 1 & -\frac{x}{c}
\end{array}\right]
$$

and, this yields an orthonormal basis for the quotient space

$$
\begin{aligned}
& J\left(X_{1}\right)=\frac{1}{c \sqrt{c^{2}+y^{2}}}\left(c^{2}+y^{2},-x y\right), \\
& J\left(X_{2}\right)=\frac{1}{\sqrt{\left(c^{2}+y^{2}\right)\left(c^{2}+x^{2}+y^{2}\right)}}\left(0, c^{2}+x^{2}+y^{2}\right) .
\end{aligned}
$$

From the orthonormal basis $\left\{J\left(X_{1}\right), J\left(X_{2}\right)\right\}$ for the quotient space, or from $\left\{X_{1}, X_{2}\right\}$ together with Formula $(0.2)$, we obtain: 

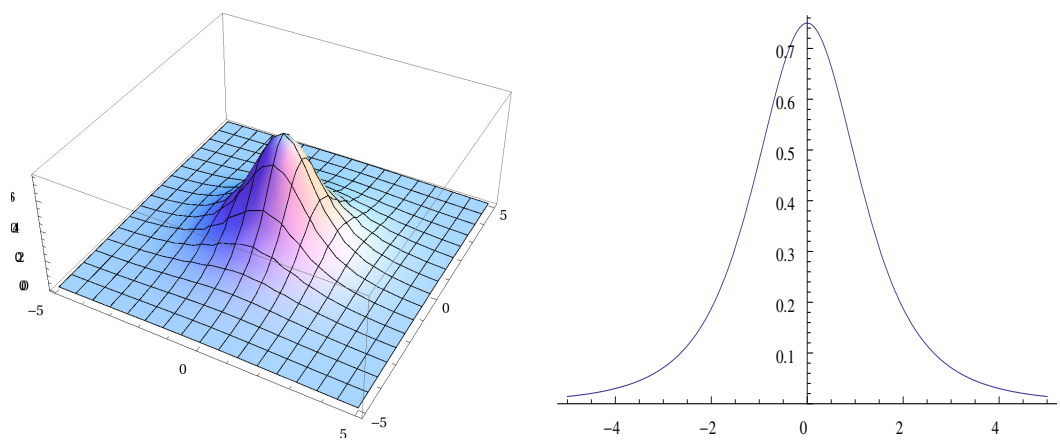

FigURE 1. $\kappa(x, y)$ of $\xi \backslash \mathbb{R}^{3}$ with $\xi(t)=\left(t c \mathbf{e}_{3}, \widehat{R}(t)\right), c=2$ and a cross section at $y=0$.

Theorem 1.3. Let $\xi: \mathbb{R} \rightarrow \mathbb{R}^{3} \rtimes \mathrm{SO}(3)=\operatorname{Isom}_{0}\left(\mathbb{R}^{3}\right)$ be a free, proper action of 1-dimensional subgroup $G$ of isometries of $\mathbb{R}^{3}$. Let

$$
\mathbb{R} \longrightarrow \mathbb{R}^{3} \longrightarrow G \backslash \mathbb{R}^{3}
$$

be the Riemannian submersion given by the action of $\xi$. The curvature $\kappa$ of the quotient space $\xi \backslash \mathbb{R}^{3}$ is as follows:

(1) $\xi$ is a pure translation : $\kappa=0$, and $G \backslash \mathbb{R}^{3}$ is isometric to the flat $\mathbb{R}^{2}$.

(2) $\xi(t)=\left(t c \mathbf{e}_{3}, \widehat{R}(t)\right), c \neq 0: N_{0}=\mathbb{R} \times \mathrm{SO}(2)$ so that $\operatorname{Isom}_{0}\left(\mathbb{R}^{3} / G\right)=$ $\mathrm{SO}(2)$, and

$$
\kappa(x, y)=\frac{3 c^{2}}{\left(c^{2}+x^{2}+y^{2}\right)^{2}} .
$$

Remark 1.4. In the case of $\xi(t)=\left(t c \mathbf{e}_{3}, \widehat{R}(t)\right), c \neq 0$ : The curvature is strictly positive; $\lim _{\|(x, y)\| \rightarrow \infty} \kappa(x, y)=0$; the maximum occurs at $(x, y)=(0,0)$ with $\kappa(0,0)=\frac{3}{c^{2}}$. As $c \rightarrow \infty, \kappa(0,0) \rightarrow 0$, and the space becomes closer to the flat $\mathbb{R}^{2}$. When $c \rightarrow 0, \kappa(0,0) \rightarrow \infty$. If $c=0$, the action is not free any more.

\section{The Heisenberg geometry Nil}

2.1. We use the global trivialization $\varphi: \mathbb{R}^{3} \rightarrow \mathrm{Nil}$

$$
\varphi(x, y, z)=\left[\begin{array}{ccc}
1 & x & z \\
0 & 1 & y \\
0 & 0 & 1
\end{array}\right] \in \mathrm{Nil}
$$

The standard left-invariant metric is given by the orthonormal basis

$$
E_{1}=\left[\begin{array}{lll}
0 & 1 & 0 \\
0 & 0 & 0 \\
0 & 0 & 0
\end{array}\right], \quad E_{2}=\left[\begin{array}{lll}
0 & 0 & 0 \\
0 & 0 & 1 \\
0 & 0 & 0
\end{array}\right], \quad E_{3}=\left[\begin{array}{lll}
0 & 0 & 1 \\
0 & 0 & 0 \\
0 & 0 & 0
\end{array}\right] \in \text { nil } .
$$


With the trivialization $\varphi$, this left-invariant metric for Nil yields a Riemannian metric on $\mathbb{R}^{3}$ which has an orthonormal basis

$$
e_{1}=\frac{\partial}{\partial x}, \quad e_{2}=\frac{\partial}{\partial y}+x \frac{\partial}{\partial z}, \quad e_{3}=\frac{\partial}{\partial z}
$$

In other words, our space Nil is isometric to $\mathbb{R}^{3}$ with the orthonormal basis $\left\{e_{1}, e_{2}, e_{3}\right\}$ at $(x, y, z)$.

2.2. With respect to this (in fact, any) left-invariant metric for Nil, the group of isometries is $\operatorname{Isom}_{0}(\mathrm{Nil})=\mathrm{Nil} \rtimes \mathrm{SO}(2) \subset \mathrm{Nil} \rtimes \operatorname{Aut}(\mathrm{Nil})$. A matrix $U=$ $\left(u_{i j}\right) \in \mathrm{GL}(2, \mathbb{R})$ becomes an automorphism of Nil by

$$
U(\varphi(x, y, z))=\varphi\left(u_{11} x+u_{12} y, u_{21} x+u_{22} y, z^{\prime}\right)
$$

where

$$
z^{\prime}=\left(u_{11} u_{22}-u_{12} u_{21}\right) z+u_{12} u_{21} x y+\frac{1}{2} u_{11} u_{21} x^{2}+\frac{1}{2} u_{12} u_{22} y^{2}
$$

and $(\mathbf{a}, U) \in \mathrm{Nil} \rtimes \mathrm{SO}(2)$ acts on $\mathbf{x} \in \mathrm{Nil}$ by

$$
(\mathbf{a}, U) \mathbf{x}=\mathbf{a} \cdot U(\mathbf{x}) .
$$

Proposition 2.3. A 1-dimensional closed subgroup $G$ of $\mathrm{Nil} \rtimes \mathrm{SO}(2)$ acting freely on $\mathrm{Nil}$ is either $\xi(t)=\left(e^{t E_{3}}, I_{2}\right), \xi(t)=\left(e^{t E_{1}}, I_{2}\right)$, or $\xi(t)=\left(e^{t c E_{3}}, R(t)\right)$ (with $c \neq 0)$ up to conjugation.

Proof. Suppose $G$ is given by a homomorphism $\xi=(\lambda, A): \mathbb{R} \rightarrow \operatorname{Nil} \rtimes \mathrm{SO}(2)$.

Assume $A$ is trivial. Then $\xi$ is of the form $\xi(t)=\left(\lambda(t), I_{2}\right)$. Let $\lambda(1)=$ $\varphi(u, v, w)=\mathbf{a}$. There are two cases: tion.

(1) If $(u, v)=(0,0)$, then $w \neq 0$, and $\xi(t)=\left(e^{t E_{3}}, I_{2}\right)$ up to reparametriza-

(2) If $(u, v) \neq(0,0)$, then, by taking $t_{0}=\arcsin \frac{-v}{\sqrt{u^{2}+v^{2}}}$,

$$
\left(1, R\left(t_{0}\right)\right)\left(\mathbf{a}, I_{2}\right)\left(1, R\left(t_{0}\right)\right)^{-1}=\left(\varphi\left(\sqrt{u^{2}+v^{2}}, 0, *\right), I_{2}\right) .
$$

By a conjugation by an element of Nil, this can be made to $\left(\varphi\left(\sqrt{u^{2}+v^{2}}, 0,0\right), I_{2}\right)$. Consequently, up to reparametrization, $\xi(t)=\left(e^{t E_{1}}, I_{2}\right)$.

(3) Assume $A$ is non-trivial so that $\xi(t)=(\lambda(t), R(t))$. Let $\xi(1)=(\lambda(1), R(1))$ $=(\mathbf{a}, U) \in \mathrm{Nil} \rtimes \mathrm{SO}(2)$. Then $U \neq I_{2}$. For $\mathbf{x} \in \mathrm{Nil}$,

$$
(\mathbf{x}, I)(\mathbf{a}, U)(\mathbf{x}, I)^{-1}=\left(\mathbf{x} \cdot \mathbf{a} \cdot U(\mathbf{x})^{-1}, U\right) .
$$

If $\mathbf{a}=\varphi(u, v, w)$, then

$$
\mathbf{x}=\varphi\left(\frac{1}{2}\left(v \cot \frac{1}{2}-u\right), \frac{1}{2}\left(-u \cot \frac{1}{2}-v\right), 0\right)
$$

will make

$$
\mathbf{x} \cdot \mathbf{a} \cdot U(\mathbf{x})^{-1}=\varphi\left(0,0, \frac{1}{4}\left(-2 u v+4 w+\left(u^{2}+v^{2}\right) \cot \frac{1}{2}\right)\right)=e^{c E_{3}}
$$

for some $c \in \mathbb{R}$. Consequently, conjugation of $(\lambda(t), R(t))$ by our $(\mathbf{x}, I)$ is $\left(e^{t c E_{3}}, R(t)\right)$. 

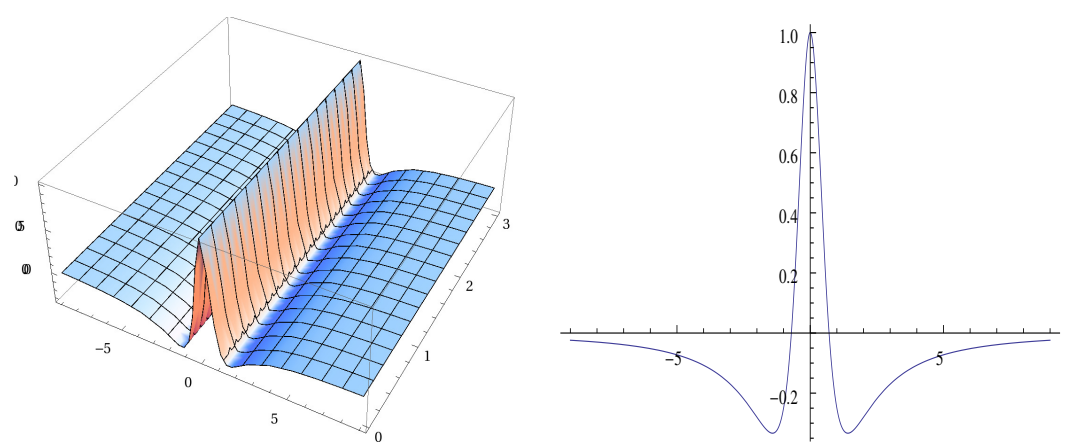

Figure 2. $\kappa(y, z)$ of $\xi \backslash$ Nil with $\xi(t)=\left(e^{t E_{1}}, I\right)$ and a cross section at $y=0$.

2.4. We shall study the Riemannian submersion of the homogeneous foliation defined by these 1-dimensional subgroups of $\operatorname{Isom}_{0}(\mathrm{Nil})$.

2.4.1 $\left(\xi(t)=\left(e^{t E_{3}}, I_{2}\right)\right)$.

The action by $\xi(t)$ is as left (or right) translation. $\xi(t) \cdot \varphi(x, y, z)=\varphi(x, y, z+$ $t)$. We make calculations on $\mathbb{R}^{3}$. So, the leaf of our foliation passing through $(x, y, z)$ is

$$
\widehat{\xi}(t)=(x, y, z+t)
$$

and the vertical vector at $(x, y, z) \in \mathbb{R}^{3}$ is

$$
V_{(x, y, z)}=\left.\frac{d}{d t}\right|_{t=0} \widehat{\xi}(t)=(0,0,1) .
$$

Let

$$
\begin{aligned}
& X_{1}=(1,0,0), \\
& X_{2}=(0,1, x) .
\end{aligned}
$$

Then $\left\{X_{1}, X_{2}\right\}$ forms an orthonormal basis of the horizontal distribution. Since $V_{(x, y, z)}=(0,0,1)$, we can take the $x y$-plane as a cross section.

The equation of leaves $(x, y, z+t)$ yields the Jacobian of the Riemannian projection on the $x y$-plane

$$
J=\left[\begin{array}{lll}
1 & 0 & 0 \\
0 & 1 & 0
\end{array}\right]
$$

and, this yields an orthonormal basis for the quotient space

$$
\begin{aligned}
& J\left(X_{1}\right)=\left(\begin{array}{ll}
1, & 0
\end{array}\right), \\
& J\left(X_{2}\right)=\left(\begin{array}{ll}
0, & 1
\end{array}\right) .
\end{aligned}
$$

The orthonormal basis $\left\{J\left(X_{1}\right), J\left(X_{2}\right)\right\}$ for the quotient space shows that the quotient space is flat. 
2.4.2 $\left(\xi(t)=\left(e^{t E_{1}}, I_{2}\right)\right)$.

The action by $\xi(t)$ is as left translation. $\xi(t) \cdot \varphi(x, y, z)=\varphi(x+t, y, z+t y)$. We make calculations on $\mathbb{R}^{3}$. So, the leaf of our foliation passing through $(x, y, z)$ is

$$
\widehat{\xi}(t)=(x+t, y, z+t y)
$$

and the vertical vector at $(x, y, z) \in \mathbb{R}^{3}$ is

$$
V_{(x, y, z)}=\left.\frac{d}{d t}\right|_{t=0} \widehat{\xi}(t)=(1,0, y) .
$$

Let

$$
\begin{aligned}
& X_{1}=\frac{1}{\sqrt{1+y^{2}}}(-y, 0,1), \\
& X_{2}=(0,-1,-x) .
\end{aligned}
$$

Then $\left\{X_{1}, X_{2}\right\}$ forms an orthonormal basis of the horizontal distribution. Since $V_{(x, y, z)}=(1,0, y)$, we can take the $y z$-plane as a cross section.

The equation of leaves $(x+t, y, z+t y)$ yields the Jacobian of the Riemannian projection on the $y z$-plane

$$
J=\left[\begin{array}{rrr}
0 & 1 & 0 \\
-y & 0 & 1
\end{array}\right]
$$

and, this yields an orthonormal basis for the quotient space

$$
\begin{aligned}
& J\left(X_{1}\right)=\left(0, \sqrt{y^{2}+1}\right), \\
& J\left(X_{2}\right)=(-1,-x) .
\end{aligned}
$$

From the orthonormal basis $\left\{J\left(X_{1}\right), J\left(X_{2}\right)\right\}$ for the quotient space, or from $\left\{X_{1}, X_{2}\right\}$ together with Formula $(0.2)$, we obtain $\kappa(y, z)=\frac{1-2 y^{2}}{\left(1+y^{2}\right)^{2}}$.

2.4.3 $\left(\xi(t)=\left(e^{t c E_{3}}, R(t)\right)\right)$.

$$
\xi(t) \cdot(x, y, z)
$$

$=\varphi\left(x \cos t-y \sin t, x \sin t+y \cos t, z+c t-x y \sin ^{2} t+\frac{1}{2}\left(x^{2}-y^{2}\right) \sin t \cos t\right)$.

We make calculations on $\mathbb{R}^{3}$. So, the leaf of our foliation passing through $(x, y, z)$ is

$\widehat{\xi}(t)=\left(x \cos t-y \sin t, x \sin t+y \cos t, z+c t-x y \sin ^{2} t+\frac{1}{2}\left(x^{2}-y^{2}\right) \sin t \cos t\right)$

and the vertical vector at $(x, y, z) \in \mathbb{R}^{3}$ is

Let

$$
V_{(x, y, z)}=\left.\frac{d}{d t}\right|_{t=0} \widehat{\xi}(t)=\left(-y, x, c+\frac{1}{2}\left(x^{2}-y^{2}\right)\right) .
$$

$$
\begin{aligned}
& X_{1}=\frac{1}{\sqrt{\left(-2 c+x^{2}+y^{2}\right)^{2}+4 y^{2}}}\left(-2 c+x^{2}+y^{2}, 0,-2 y\right), \\
& X_{2}=\frac{1}{D}\left(-4 x y,-\left(-2 c+x^{2}+y^{2}\right)^{2}-4 y^{2}, E\right),
\end{aligned}
$$



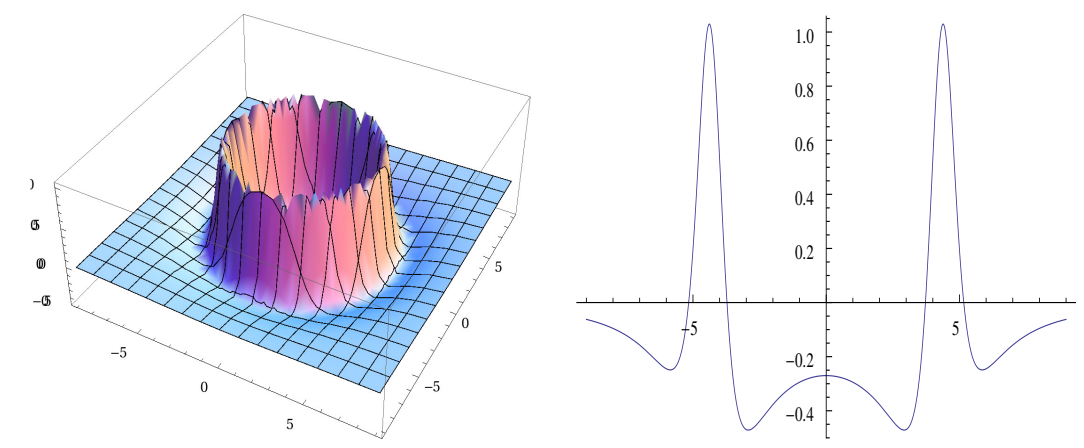

Figure 3. $\kappa(x, y)$ of $\xi \backslash$ Nil with $\xi(t)=\left(e^{t c E_{3}}, R(t)\right), c=2$, and a cross section at $y=0$.

where $D=\sqrt{\left(\left(-2 c+x^{2}+y^{2}\right)^{2}+4 y^{2}\right)\left(\left(-2 c+x^{2}+y^{2}\right)^{2}+4\left(x^{2}+y^{2}\right)\right)}$ and $E=-x\left(4 c^{2}-4 c\left(x^{2}+y^{2}+1\right)+\left(x^{2}+y^{2}\right)^{2}+2\left(x^{2}+3 y^{2}\right)\right)$. Then $\left\{X_{1}, X_{2}\right\}$ form an orthogonal basis of the horizontal distribution.

If $c=0$, the action is not free. We take the orbit of the line $\{(x, y): x \in$ $[0, \infty), y=0\}$ by the action of $\mathrm{SO}(2) \subset \operatorname{Aut}(\mathrm{Nil})$,

$$
\begin{aligned}
\mathcal{S} & =\left\{\left(x \cos t, x \sin t, \frac{1}{2} x^{2} \sin t \cos t\right)\right\} \\
& =\left\{(x, y, z): \quad z=\frac{1}{2} x y\right\}
\end{aligned}
$$

as a global cross section. The equation of leaves $\widehat{\xi}(t)$ yields the Jacobian of the Riemannian projection on $\mathcal{S}$.

$$
J=\left[\begin{array}{ccc}
1-\frac{y^{2}}{2 c} & -\frac{x y}{2 c} & \frac{y}{c} \\
\frac{x y}{2 c} & \frac{x^{2}}{2 c}+1 & -\frac{x}{c}
\end{array}\right]
$$

and, this yields an orthonormal basis for the quotient space

$$
\begin{aligned}
& J\left(X_{1}\right)=\frac{1}{D}\left(-4 c^{2}+2\left(x^{2}+2 y^{2}\right) c-y^{2}\left(x^{2}+y^{2}+4\right), x y\left(x^{2}+y^{2}-2 c+4\right)\right), \\
& J\left(X_{2}\right)=\frac{E}{D}\left(-x y, x^{2}-2 c\right),
\end{aligned}
$$

where $D=2 c \sqrt{4 y^{2}+\left(x^{2}+y^{2}-2 c\right)^{2}}, E=\sqrt{\left(x^{2}+y^{2}-2 c\right)^{2}+4\left(x^{2}+y^{2}\right)}$. From this orthonormal basis $\left\{J\left(X_{1}\right), J\left(X_{2}\right)\right\}$ for the quotient space, or from $\left\{X_{1}, X_{2}\right\}$ together with Formula (0.2), we obtain

$$
\kappa(x, y)=\frac{2\left(-24 c^{3}+4 c^{2}\left(5 x^{2}+5 y^{2}+6\right)-2 c\left(x^{2}+y^{2}\right)^{2}-\left(x^{2}+y^{2}-2\right)\left(x^{2}+y^{2}\right)^{2}\right)}{\left(\left(-2 c+x^{2}+y^{2}\right)^{2}+4\left(x^{2}+y^{2}\right)\right)^{2}} .
$$

Summarizing all, we have: 
Theorem 2.5. Let $\xi: \mathbb{R} \rightarrow \mathrm{Nil} \rtimes \mathrm{SO}(2)=\mathrm{Isom}_{0}(\mathrm{Nil})$ be a free, proper action of 1-dimensional subgroup of isometries of Nil. Let

$$
\mathbb{R} \longrightarrow \mathrm{Nil} \longrightarrow \xi \backslash \mathrm{Nil}
$$

be the Riemannian submersion given by the action of $\xi$. The curvature $\kappa$ of the quotient space $\xi \backslash \mathrm{Nil}$ is as follows:

(1) $\xi(t)=\left(e^{t E_{3}}, I_{2}\right): \kappa(x, y)=0$, and $\xi \backslash \mathrm{Nil}$ is isometric to the flat $\mathbb{R}^{2}$.

(2) $\xi(t)=\left(e^{t E_{1}}, I_{2}\right): \kappa(y, z)=\frac{1-2 y^{2}}{\left(1+y^{2}\right)^{2}}$ and $\operatorname{Isom}_{0}(\xi \backslash \mathrm{Nil})=\left\{\exp t E_{3}\right\} \cong$ $\mathbb{R}$, translations in the central direction.

(3) $\xi(t)=\left(e^{t c E_{3}}, R(t)\right)$ :

$$
\kappa(x, y)=\frac{2\left(-24 c^{3}+4 c^{2}\left(5 r^{2}+6\right)-2 c r^{4}-\left(r^{2}-2\right) r^{4}\right)}{\left(\left(r^{2}-2 c\right)^{2}+4 r^{2}\right)^{2}},
$$

where $r^{2}=x^{2}+y^{2}$. $\operatorname{Isom}_{0}(\xi \backslash \mathrm{Nil})=\mathrm{SO}(2)$ is generated by the rotations $(1, R(t))$.

Remark 2.6. (2) $\kappa(y, z)$ has values in $-\frac{1}{3} \leq \kappa \leq 1$ with critical values $\kappa(0, z)=$ $1, \kappa( \pm \sqrt{2}, z)=-\frac{1}{3} ;$ and $\lim _{y \rightarrow \infty} \kappa(y, z)=0$.

(3) $\xi(t)=\left(e^{t c E_{3}}, R(t)\right): \kappa(0,0)=\frac{3(1-c)}{c^{2}}$ and $\lim _{\|(x, y)\| \rightarrow \infty} \kappa(x, y)=0$. As $c \rightarrow \infty, \kappa(0,0) \rightarrow 0$, and the space becomes closer to the flat $\mathbb{R}^{2}$. When $c \rightarrow 0$, $\kappa(0,0) \rightarrow \infty$.

\section{3. $S^{2} \times \mathbb{R}$}

3.1. The group of isometries of $S^{2} \times \mathbb{R}$ is $\operatorname{Isom}_{0}\left(S^{2} \times \mathbb{R}\right)=\mathrm{SO}(3) \times \mathbb{R}$. A 1-dimensional closed subgroup of $\mathrm{SO}(3) \times \mathbb{R}$ which can act freely on $S^{2} \times \mathbb{R}$ is $\xi(t)=(A(t), c t)$. If $A(t)$ is trivial, then $\xi(t)$ is a pure translation to the $\mathbb{R}$-factor, and the orbit space $\mathbb{R} \backslash\left(S^{2} \times \mathbb{R}\right)=S^{2}$ as a Riemannian manifold. Otherwise,

$$
\xi(t)=(\widehat{R}(t), c t)
$$

since $A(t)$ must be in a maximal torus (i.e., every other closed 1-dimensional subgroup of $\mathrm{SO}(3)$ is a conjugate of $\widehat{R}(t))$.

Give a coordinate system on $S^{2} \times \mathbb{R}, \varphi: \mathbb{R}^{3} \rightarrow S^{2} \times \mathbb{R}$, defined by

$$
\varphi(x, y, z)=\left(x, y, \sqrt{1-x^{2}-y^{2}}, z\right) \in S^{2} \times \mathbb{R} \subset \mathbb{R}^{3} \times \mathbb{R}
$$

(in fact, only on $\left(S^{2}\right)^{+} \times \mathbb{R}$ ).

3.2. An orthonormal basis at $(x, y, u, z)$, where $u=\sqrt{1-x^{2}-y^{2}}$,

$$
\begin{aligned}
& E_{1}=\frac{1}{\sqrt{x^{2}+y^{2}}}(-y, x, 0,0), \\
& E_{2}=\frac{u}{\sqrt{x^{2}+y^{2}}}\left(-x,-y, \frac{x^{2}+y^{2}}{u}, 0\right), \\
& E_{3}=(0,0,0,1),
\end{aligned}
$$


translate to an orthonormal basis at $\mathbb{R}^{3}$ by $\varphi_{*}^{-1}$ to

$$
\begin{aligned}
& e_{1}=\frac{1}{\sqrt{x^{2}+y^{2}}}(-y, x, 0), \\
& e_{2}=\frac{u}{\sqrt{x^{2}+y^{2}}}(-x,-y, 0), \\
& e_{3}=(0,0,1) .
\end{aligned}
$$

3.3. For our $\xi(t)=(\widehat{R}(t), c t)$,

$$
\xi(t) \cdot(x, y, u, z)=((\cos t) x-(\sin t) y,(\sin t) x+(\cos t) y, u, z+c t) .
$$

The vertical vector $\left.\frac{d}{d t}\right|_{t=0} \xi(t) \cdot(x, y, u, z)=(-y, x, 0, c)$ translates to

$$
V_{(x, y, z)}=(-y, x, c)
$$

on $\mathbb{R}^{3}$. Clearly, $S^{2} \times\{0\}$ is a global cross section for the Riemannian submersion $\left(S^{2} \times \mathbb{R}\right) \rightarrow \xi \backslash\left(S^{2} \times \mathbb{R}\right)$. Let

$$
\begin{aligned}
& X_{1}=\frac{1}{\sqrt{\left(x^{2}+y^{2}\right)\left(c^{2}+x^{2}+y^{2}\right)}}\left(c y,-c x, x^{2}+y^{2}\right), \\
& X_{2}=\frac{\sqrt{1-x^{2}-y^{2}}}{\sqrt{x^{2}+y^{2}}}(x, y, 0) .
\end{aligned}
$$

Then $\left\{X_{1}, X_{2}\right\}$ forms an orthonormal basis of the horizontal distribution.

The equation of leaves $((\cos t) x-(\sin t) y,(\sin t) x+(\cos t) y, u, z+c t)$ yields the Jacobian of the Riemannian projection on the $x y$-plane

$$
J=\left[\begin{array}{rrr}
1 & 0 & \frac{y}{c} \\
0 & 1 & -\frac{x}{c}
\end{array}\right]
$$

and, this yields an orthonormal basis for the quotient space

$$
\begin{aligned}
& J\left(X_{1}\right)=\frac{\sqrt{c^{2}+x^{2}+y^{2}}}{c \sqrt{x^{2}+y^{2}}}(y,-x), \\
& J\left(X_{2}\right)=\frac{\sqrt{1-x^{2}-y^{2}}}{\sqrt{x^{2}+y^{2}}}(x, y) .
\end{aligned}
$$

From this orthonormal basis $\left\{J\left(X_{1}\right), J\left(X_{2}\right)\right\}$ for the quotient space, or from $\left\{X_{1}, X_{2}\right\}$ together with Formula $(0.2)$, we obtain

$$
\kappa(x, y)=\frac{c^{2}\left(c^{2}-2 x^{2}-2 y^{2}+3\right)}{\left(c^{2}+x^{2}+y^{2}\right)^{2}} .
$$

Theorem 3.4. Let $\xi(t)=(A(t), c t): \mathbb{R} \rightarrow \mathrm{SO}(3) \times \mathbb{R}=\operatorname{Isom}_{0}\left(S^{2} \times \mathbb{R}\right)$ be a free, proper action of 1-dimensional subgroup of isometries of $S^{2} \times \mathbb{R}$. Let

$$
\mathbb{R} \rightarrow S^{2} \times \mathbb{R} \rightarrow \xi \backslash\left(S^{2} \times \mathbb{R}\right)
$$

be the Riemannian submersion given by the action of $\xi$. The curvature $\kappa$ of the quotient space $\xi \backslash\left(S^{2} \times \mathbb{R}\right)$ is as follows: 

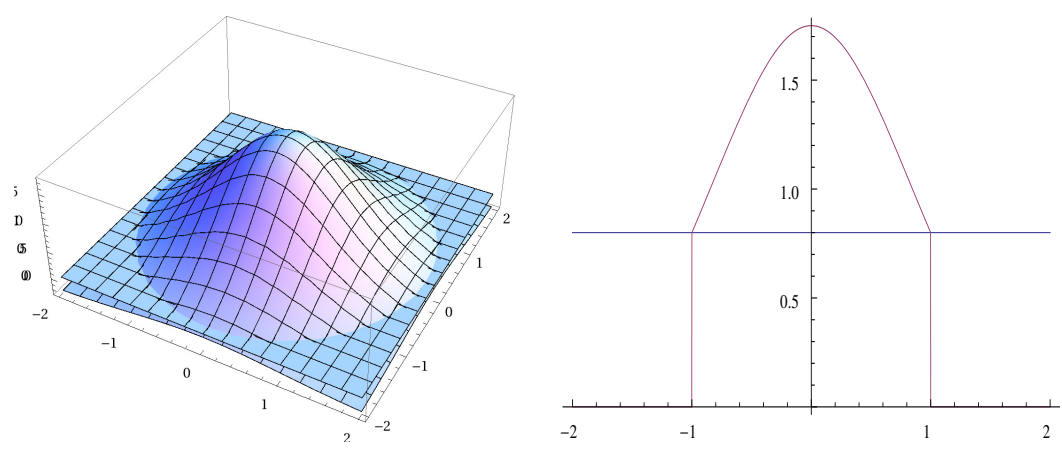

FiguRE 4. $\kappa(x, y)$ of $\xi \backslash\left(S^{2} \times \mathbb{R}\right)$ with $\xi(t)=(R(t), c t), c=2$, and a cross section at $y=0$.

(1) If $A(t)$ is trivial, then $\xi(t)=(I, t), \xi \backslash\left(S^{2} \times \mathbb{R}\right)$ is the standard $S^{2}$ of curvature +1 .

(2) If $\xi(t)=(\widehat{R}(t), c t), c \neq 0$, then

$$
\kappa(x, y, \pm u)=\frac{c^{2}\left(c^{2}-2 x^{2}-2 y^{2}+3\right)}{\left(c^{2}+x^{2}+y^{2}\right)^{2}},
$$

where $(x, y, \pm u) \in S^{2}$. The normalizer of $\xi$ is $N_{0}=\mathrm{SO}(2) \times \mathbb{R}$, and $\operatorname{Isom}_{0}\left(\xi \backslash S^{2} \times\right.$ $\mathbb{R})=\mathrm{SO}(2)$.

Remark 3.5. For $\xi(t)=(R(t), c t), \kappa$ attains its maximum $1+\frac{3}{c^{2}}$ at $(0,0, \pm 1)$, and its minimum $\frac{c^{2}}{c^{2}+1}$ on the equator $x^{2}+y^{2}=1$ so that $\frac{c^{2}}{c^{2}+1} \leq \kappa \leq 1+\frac{3}{c^{2}}$. If $c \rightarrow \infty$, the situation becomes closer to the case $\xi(t)=(I, t)$.

\section{The sphere $S^{3}$}

The isometry group is $\operatorname{Isom}_{0}\left(S^{3}\right)=\mathrm{SO}(4)$. It is known [1] that the only free and proper $S^{1}$-action on $S^{3}$ is the Hopf fibration $S^{1} \rightarrow S^{3} \rightarrow S^{2}\left(\frac{1}{2}\right)$, up to orthogonal congruence. Therefore the quotient space $S^{2}\left(\frac{1}{2}\right)$ has constant curvature $\kappa=4$.

\section{5. $\mathbb{H}^{2} \times \mathbb{R}$}

5.1. The space $\mathbb{H}^{2} \times \mathbb{R}=\{(x, y, z) \mid y>0\}$ has a metric $d s^{2}=\frac{d x^{2}+d y^{2}}{y^{2}}+d z^{2}$, and the group of isometries is $\operatorname{Isom}_{0}\left(\mathbb{H}^{2} \times \mathbb{R}\right)=\mathrm{PSL}_{2} \mathbb{R} \times \mathbb{R}$. A 1-dimensional subgroup of $\mathrm{PSL}_{2} \mathbb{R} \times \mathbb{R}$ is of the form

$$
\xi(t)=(\zeta(t), c t) \in \mathrm{PSL}_{2} \mathbb{R} \times \mathbb{R} .
$$

There are 4 distinct cases for $\zeta(t)$ : trivial, parabolic, hyperbolic, and elliptic.

5.1.1 $(\zeta(t)$ is trivial).

The orbit space by $\xi$ is the genuine $\mathbb{H}^{2}$. 

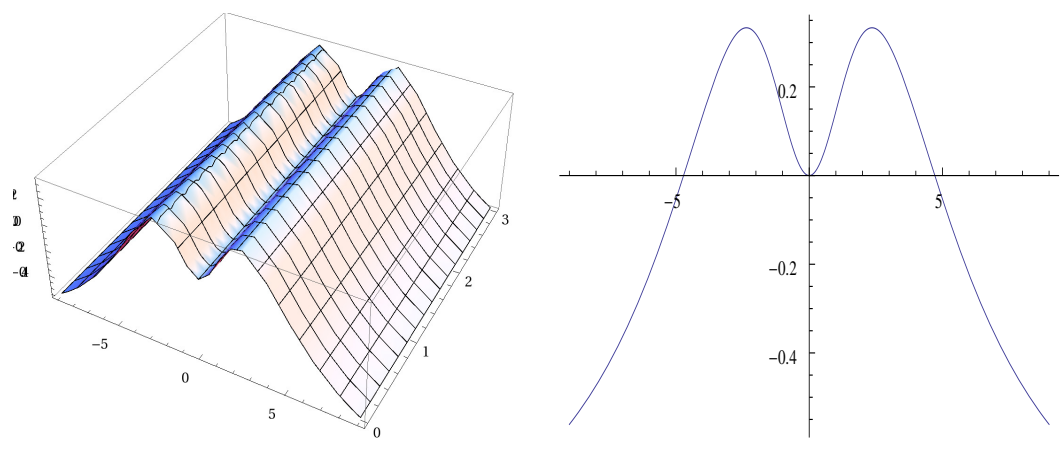

FiguRE 5. $\kappa(y, z)$ of $\xi \backslash\left(\mathbb{H}^{2} \times \mathbb{R}\right)$ with $\xi(t)=(($ parabolic $), c t)$, $c=0.3, z=0$.

5.1.2 $\left(\zeta(t)=\exp \left(t\left[\begin{array}{ll}0 & 1 \\ 0 & 0\end{array}\right]\right)=\left[\begin{array}{ll}1 & t \\ 0 & 1\end{array}\right](\right.$ parabolic $\left.)\right)$.

In this case,

$$
\xi(t) \cdot(x, y, z)=(x+t, y, z+c t)
$$

so that the vertical vector is $V_{(x, y, z)}=(1,0, c)$, and

$$
\begin{aligned}
& X_{1}=\frac{1}{\sqrt{1+c^{2} y^{2}}}\left(-c y^{2}, 0,1\right), \\
& X_{2}=(0,-y, 0),
\end{aligned}
$$

are an orthonormal basis of the horizontal distribution. Clearly, the $y z$-plane is a global cross section. The equation of leaves $(x+t, y, z+c t)$ yields the Jacobian of the Riemannian projection on the $y z$-plane

$$
J=\left[\begin{array}{rrr}
0 & 1 & 0 \\
-c & 0 & 1
\end{array}\right]
$$

and, this yields an orthonormal basis for the quotient space

$$
\begin{aligned}
& J\left(X_{1}\right)=\left(0, \sqrt{c^{2} y^{2}+1}\right), \\
& J\left(X_{2}\right)=(-y, 0) .
\end{aligned}
$$

From this orthonormal basis $\left\{J\left(X_{1}\right), J\left(X_{2}\right)\right\}$ for the quotient space, or from $\left\{X_{1}, X_{2}\right\}$ together with Formula (0.2), we obtain

$$
\kappa(y, z)=\frac{c^{2} y^{2}\left(2-c^{2} y^{2}\right)}{\left(1+c^{2} y^{2}\right)^{2}} .
$$

Since there is an isometry translating to the $z$-direction, $\kappa(y, 0)$ is enough, but $\kappa(y, z)$ is independent of $z$ already. Note that $\lim _{y \rightarrow \infty} \kappa(y, z)=-1$.

5.1.3 $\left(\zeta(t)=\exp \left(t\left[\begin{array}{cc}1 & 0 \\ 0 & -1\end{array}\right]\right)=\left[\begin{array}{cc}e^{t} & 0 \\ 0 & e^{-t}\end{array}\right]\right.$ (hyperbolic) $)$.

In this case,

$$
\xi(t) \cdot(x, y, z)=\left(e^{2 t} x, e^{2 t} y, z+c t\right)
$$



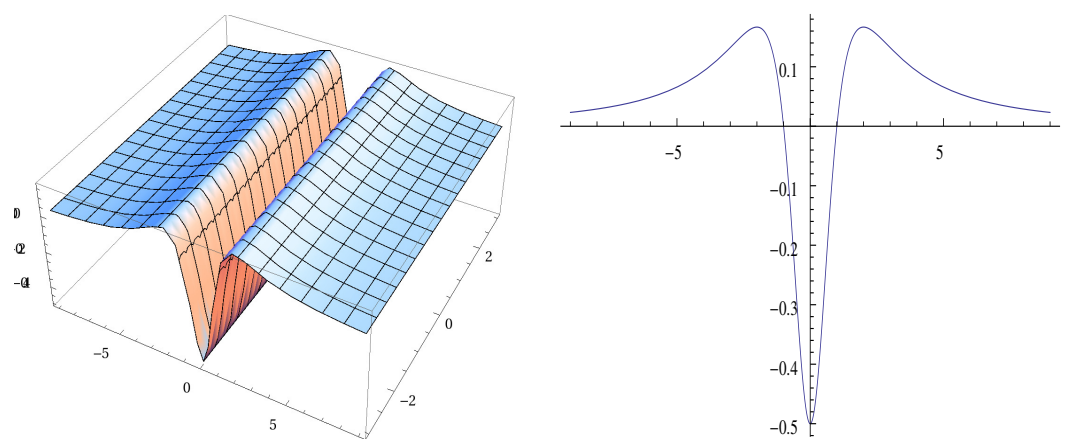

FiguRE $6 . \kappa(x, z)$ of $\xi \backslash\left(\mathbb{H}^{2} \times \mathbb{R}\right)$ with $\xi(t)=(($ hyperbolic $), c t)$, $c=2, z=0$.

so that the vertical vector is $V_{(x, y, z)}=\frac{y}{\sqrt{c^{2}+4 x^{2}+4}}(2 x, 2 y, c)$ and

$$
\begin{aligned}
& X_{1}=\frac{1}{\sqrt{4 x^{2}+c^{2} y^{2}}}\left(-c y^{2}, 0,2 x\right), \\
& X_{2}=\frac{y}{\sqrt{\left(4 x^{2}+c^{2} y^{2}\right)\left(4 x^{2}+\left(4+c^{2}\right) y^{2}\right)}}\left(4 x y,-\left(4 x^{2}+c^{2} y^{2}\right), 2 c y\right)
\end{aligned}
$$

make an orthonormal basis of the horizontal distribution.

The equation of leaves $\left(e^{2 t} x, e^{2 t} y, z+c t\right)$ yields the Jacobian of the Riemannian projection on the $x z$-plane (with $y=1$ )

$$
J=\left[\begin{array}{lll}
1 & -x & 0 \\
0 & -\frac{c}{2} & 1
\end{array}\right]
$$

and, this yields an orthonormal basis for the quotient space

$$
\begin{aligned}
& J\left(X_{1}\right)=\frac{1}{\sqrt{c^{2}+4 x^{2}}}(-c, 2 x), \\
& J\left(X_{2}\right)=\frac{\sqrt{c^{2}+4 x^{2}+4}}{2 \sqrt{c^{2}+4 x^{2}}}(2 x, c) .
\end{aligned}
$$

Clearly, $(x, z) \rightarrow(x, z+t)$ is an isometry. From this orthonormal basis $\left\{J\left(X_{1}\right)\right.$, $\left.J\left(X_{2}\right)\right\}$ for the quotient space, or from $\left\{X_{1}, X_{2}\right\}$ together with Formula (0.2), we obtain

$$
\kappa(x, z)=-\frac{c^{2}\left(c^{2}-8 x^{2}+4\right)}{\left(c^{2}+4 x^{2}+4\right)^{2}} .
$$

5.1.4 $\left(\zeta(t)=\exp \left(t\left[\begin{array}{cc}0 & -1 \\ 1 & 0\end{array}\right]\right)=R(t)(\right.$ elliptic $\left.)\right)$.

In this case,

$$
\xi(t) \cdot(x, y, z)=\left(\frac{1}{2 D}\left(2 x \cos 2 t+\left(-1+x^{2}+y^{2}\right) \sin 2 t\right), \frac{y}{D}, c t+z\right),
$$

where $D=\cos ^{2} t+\left(x^{2}+y^{2}\right) \sin ^{2} t+x \sin 2 t$. Then the vertical vector is

$$
V_{(x, y, z)}=\left(-1-x^{2}+y^{2},-2 x y, c\right) \text {. }
$$



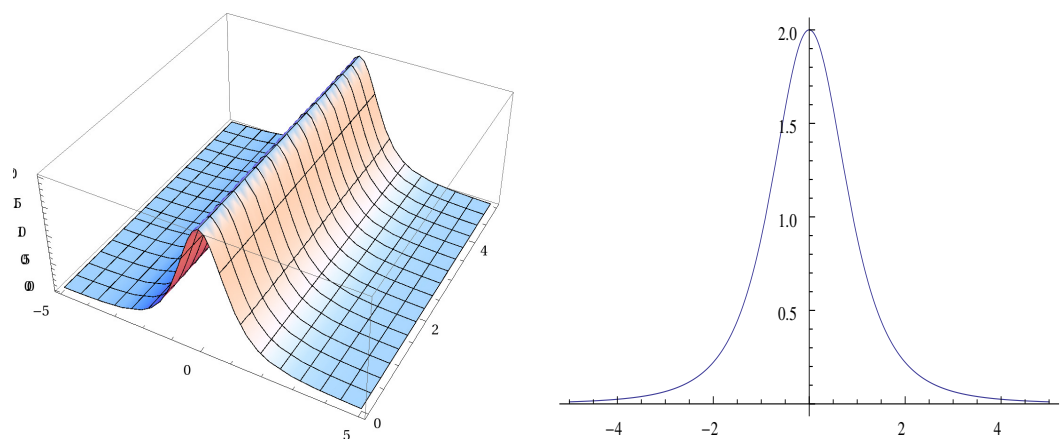

FiguRe 7. $\kappa(x, z)$ of $\xi \backslash\left(\mathbb{H}^{2} \times \mathbb{R}\right)$ with $\xi(t)=(($ elliptic $), c t)$, $c=2$, and cross-section at $z=0$.

If $c=0$, the action is not free.

We take the $x z$-plane $(y=1)$ as a global cross section, and

$$
\begin{aligned}
& X_{1}=\frac{1}{\sqrt{x^{4}+c^{2}}}\left(-c, 0,-x^{2}\right), \\
& X_{2}=\frac{1}{\sqrt{\left(x^{4}+c^{2}\right)\left(x^{4}+4 x^{2}+c^{2}\right)}}\left(2 x^{3},-x^{4}-c^{2}, 2 c x\right),
\end{aligned}
$$

make an orthonormal basis of the horizontal distribution.

The equation of leaves yields the Jacobian of the Riemannian projection on the $x z$-plane

$$
J=\left[\begin{array}{rrr}
1 & -\frac{x}{2} & 0 \\
0 & \frac{c}{2 x} & 1
\end{array}\right]
$$

and, this yields an orthonormal basis for the quotient space

$$
\begin{aligned}
& J\left(X_{1}\right)=-\frac{1}{\sqrt{x^{4}+c^{2}}}\left(c, x^{2}\right), \\
& J\left(X_{2}\right)=\frac{\sqrt{x^{4}+4 x^{2}+c^{2}}}{2 x \sqrt{x^{4}+c^{2}}}\left(x^{2},-c\right) .
\end{aligned}
$$

Clearly, $(x, z) \rightarrow(x, z+t)$ is an isometry. From this orthonormal basis $\left\{J\left(X_{1}\right)\right.$, $\left.J\left(X_{2}\right)\right\}$ for the quotient space, or from $\left\{X_{1}, X_{2}\right\}$ together with Formula (0.2), we obtain

$$
\kappa(x, z)=\frac{c^{2}\left(2\left(x^{4}+4 x^{2}+6\right)-c^{2}\right)}{\left(c^{2}+x^{4}+4 x^{2}\right)^{2}} .
$$

Theorem 5.2. Let $\xi(t)=(\zeta(t), c t) \in \mathrm{PSL}_{2} \mathbb{R} \times \mathbb{R}=\operatorname{Isom}_{0}\left(\mathbb{H}^{2} \times \mathbb{R}\right)$ be a free, proper action of 1-dimensional subgroup of isometries of $\mathbb{H}^{2} \times \mathbb{R}$. The curvature $\kappa$ of the quotient space $\xi \backslash\left(\mathbb{H}^{2} \times \mathbb{R}\right)$ is as follows:

(1) If $\zeta(t)$ is trivial, then $\xi(t)=(I, t), \xi \backslash\left(\mathbb{H}^{2} \times \mathbb{R}\right)$ is the standard $\mathbb{H}^{2}$ of curvature -1 . 
(2) $\zeta(t)=\exp \left(t\left[\begin{array}{ll}0 & 1 \\ 0 & 0\end{array}\right]\right)$ (parabolic). If $c=0, \kappa(y, z)=0$, flat. If $c \neq$ $0, \kappa(y, z)=\frac{c^{2} y^{2}\left(2-c^{2} y^{2}\right)}{\left(1+c^{2} y^{2}\right)^{2}}$ and $\operatorname{Isom}_{0}\left(\xi \backslash \mathbb{H}^{2} \times \mathbb{R}\right)=\mathbb{R}$ generated by the translation on the $\mathbb{R}$-factor.

(3) $\zeta(t)=\exp \left(t\left[\begin{array}{cc}1 & 0 \\ 0 & -1\end{array}\right]\right)$ (hyperbolic).

$\kappa(x, z)=-\frac{c^{2}\left(c^{2}-8 x^{2}+4\right)}{\left(c^{2}+4 x^{2}+4\right)^{2}}$ and $\operatorname{Isom}_{0}\left(\xi \backslash \mathbb{H}^{2} \times \mathbb{R}\right)=\mathbb{R}$ generated by the translation on the $\mathbb{R}$-factor.

(4) $\zeta(t)=\exp \left(t\left[\begin{array}{cc}0 & -1 \\ 1 & 0\end{array}\right]\right)=R(t)$ (elliptic), $(c \neq 0)$.

$\kappa(x, z)=\frac{c^{2}\left(2\left(x^{4}+4 x^{2}+6\right)-c^{2}\right)}{\left(c^{2}+x^{4}+4 x^{2}\right)^{2}}$ and $\operatorname{Isom}_{0}\left(\xi \backslash \mathbb{H}^{2} \times \mathbb{R}\right)=\mathbb{R}$ generated by the translation on the $\mathbb{R}$-factor.

Proof. In the case of $(2)$ and (3), the normalizer $N_{0}$ of $\xi$ in $\operatorname{Isom}_{0}(\mathbb{H} \times \mathbb{R})$ is $\mathbb{R} \times \mathbb{R}$, where the first $\mathbb{R}$-factor is coming from the parabolic/hyperbolic subgroup, and the second $\mathbb{R}$ is the translation in the $\mathbb{R}$-factor. The quotient is $N_{0} / \xi=\mathbb{R}$, and since the curvature is not constant, $\operatorname{Isom}_{0}\left(\xi \backslash \mathbb{H}^{2} \times \mathbb{R}\right)$ cannot be 2-dimensional. For (4), the normalizer is $N_{0}=\mathrm{SO}(2) \times \mathbb{R}$, and $\operatorname{Isom}_{0}(\xi \backslash \mathbb{H} \times$ $\mathbb{R})=(\mathrm{SO}(2) \times \mathbb{R}) / \mathrm{SO}(2)=\mathbb{R}$.

Remark 5.3. (2) Note that this quotient has 1-dimensional isometry group unless $c=0$. When $c=0$, the quotient is apparently a product $\mathbb{R} \times \mathbb{R}$ so that it is flat. In general, $\kappa$ attains its maximum value $\frac{1}{3}$ at $y=\frac{1}{\sqrt{2} c}$, and $-1<\kappa \leq \frac{1}{3}$. Further, $\lim _{y \rightarrow 0} \kappa=0$ and $\lim _{y \rightarrow \infty} \kappa=-1$.

(3) For $x=0, \kappa=-\frac{c^{2}}{4+c^{2}}$; and for $x= \pm \frac{\sqrt{4+c^{2}} y}{\sqrt{2}}, \kappa=\frac{c^{2}}{3\left(4+c^{2}\right)}$. When $c=0$, $\kappa=0$. Further, $\lim _{y \rightarrow 0} \kappa=0 ; \lim _{y \rightarrow \infty} \kappa=-\frac{c^{2}}{4+c^{2}}$.

(4) For $x=0, \kappa=\frac{12}{c^{2}}-1$; and $\kappa>0$ for all $x>0$. $\lim _{x \rightarrow \infty} \kappa=0$ and $\lim _{x \rightarrow \infty} \kappa=0$.

\section{The universal covering group of $\mathrm{PSL}_{2} \mathbb{R}$}

6.1. Let $P=\mathrm{PSL}_{2} \mathbb{R}$. Give the metric on the Lie algebra of $P$ so that

$$
E_{1}=\frac{1}{2}\left[\begin{array}{ll}
0 & 1 \\
1 & 0
\end{array}\right], E_{2}=\frac{1}{2}\left[\begin{array}{cc}
1 & 0 \\
0 & -1
\end{array}\right], E_{3}=\frac{1}{2}\left[\begin{array}{cc}
0 & -1 \\
1 & 0
\end{array}\right]
$$

are orthonormal. With this standard left-invariant Riemannian metric on $P$, the group of isometries of $P$ is $\operatorname{Isom}_{0}(P)=P \times K$, where $K$ the is maximal compact subgroup of $P$ generated by $\exp \left(t E_{3}\right)$, acting as right translations. Therefore,

$$
\operatorname{Isom}_{0}\left(\widetilde{\mathrm{PSL}_{2}} \mathbb{R}\right)=\widetilde{\mathrm{PSL}_{2}} \mathbb{R} \times_{\mathbb{Z}} \widetilde{K}
$$

where $\mathbb{Z}=\widetilde{\mathrm{PSL}_{2}} \mathbb{R} \cap \widetilde{K}$ is the center of $\widetilde{\mathrm{PSL}_{2}} \mathbb{R}$, sitting diagonally. We shall use $\widetilde{P}$ to denote $\widetilde{\mathrm{PSL}_{2}} \mathbb{R}$. Let $G$ be a 1 -dimensional subgroup of $\operatorname{Isom}(\widetilde{P})$. We study the quotient Riemannian manifolds of the various Riemannian submersions $\widetilde{P} \rightarrow G \backslash \widetilde{P}$. To do this, we will use the Iwasawa decomposition of $\mathrm{P}$; see, for example, [2]:

$$
P=N A K \quad(\text { instead of } K A N)
$$


where

$$
N=\left\{\left[\begin{array}{ll}
1 & x \\
0 & 1
\end{array}\right]\right\}, A=\left\{\left[\begin{array}{cc}
e^{y} & 0 \\
0 & e^{-y}
\end{array}\right]\right\}, K=\left\{\left[\begin{array}{cc}
\cos z & -\sin z \\
\sin z & \cos z
\end{array}\right]\right\} .
$$

If $W=\frac{1}{2}\left(E_{1}-E_{3}\right)$, then $N=e^{2 x W}$. Take the global coordinate system $\varphi$ on $\widetilde{P}$ by

$$
\begin{aligned}
\varphi(x, y, z) & =e^{2 x W} e^{2 y E_{2}} e^{2 z E_{3}} \\
& =\left[\begin{array}{ll}
1 & x \\
0 & 1
\end{array}\right]\left[\begin{array}{cc}
e^{y} & 0 \\
0 & e^{-y}
\end{array}\right]\left[\begin{array}{rr}
\cos z & -\sin z \\
\sin z & \cos z
\end{array}\right] .
\end{aligned}
$$

6.2. A 1-dimensional subgroup of $\widetilde{\mathrm{PSL}}_{2} \mathbb{R} \times_{\mathbb{Z}} \widetilde{K}$ is of the form

$$
\begin{aligned}
\xi(t) & =\left(e^{2 t\left(u W+v E_{2}+w E_{3}\right)}, 2 c t\right) \\
& =(\zeta(t), 2 c t) \quad \in \mathrm{PSL}_{2} \mathbb{R} \times \mathbb{R} .
\end{aligned}
$$

There are 4 distinct cases for $\zeta(t)$ : trivial, parabolic, hyperbolic, or elliptic, depending on $(u, v, w)=(0,0,0),(1,0,0),(0,1,0)$, or $(0,0,1)$.

6.2.1 $(\xi(t)=(\zeta(t), 2 c t)$, with $(u, v, w)=(0,0,0), c \neq 0$ (with $\zeta(t)$ trivial $))$.

The group is the right translation by $\widetilde{K}$, and the orbit space by $\xi$ is the genuine $\mathbb{H}^{2}$ (with $\kappa=-1$ ). This shows that $\mathrm{PSL}_{2} \mathbb{R}$ is the unit tangent bundle of $\mathbb{H}^{2}$.

6.2.2 $(\xi(t)=(\zeta(t), 2 c t)$, with $(u, v, w)=(1,0,0)$ (with $\zeta(t)$ parabolic $))$.

In this case,

$$
\xi(t) \cdot \varphi(x, y, z)=\varphi(x+t, y, z+c t)
$$

so that the vertical vector is $V_{(x, y, z)}=(1,0, c)$. The $y z$-plane is a global cross section.

$$
\begin{aligned}
& X_{1}=\frac{1}{\sqrt{\left(1-2 c e^{2 y}\right)^{2}+1}}\left(e^{2 y}\left(1-2 c e^{2 y}\right), 0,1-c e^{2 y}\right), \\
& \left.X_{2}=\left(0,-\frac{1}{2}, 0\right)\right),
\end{aligned}
$$

make an orthonormal basis of the horizontal distribution.

The equation of leaves $(x+t, y, z+c t)$ yields the Jacobian of the Riemannian projection on the $y z$-plane

$$
J=\left[\begin{array}{rrr}
0 & 1 & 0 \\
-c & 0 & 1
\end{array}\right]
$$

and, this yields an orthonormal basis for the quotient space

$$
\begin{aligned}
& J\left(X_{1}\right)=\left(0, \sqrt{c e^{2 y}\left(c e^{2 y}-1\right)+\frac{1}{2}}\right), \\
& J\left(X_{2}\right)=\left(-\frac{1}{2}, 0\right) .
\end{aligned}
$$



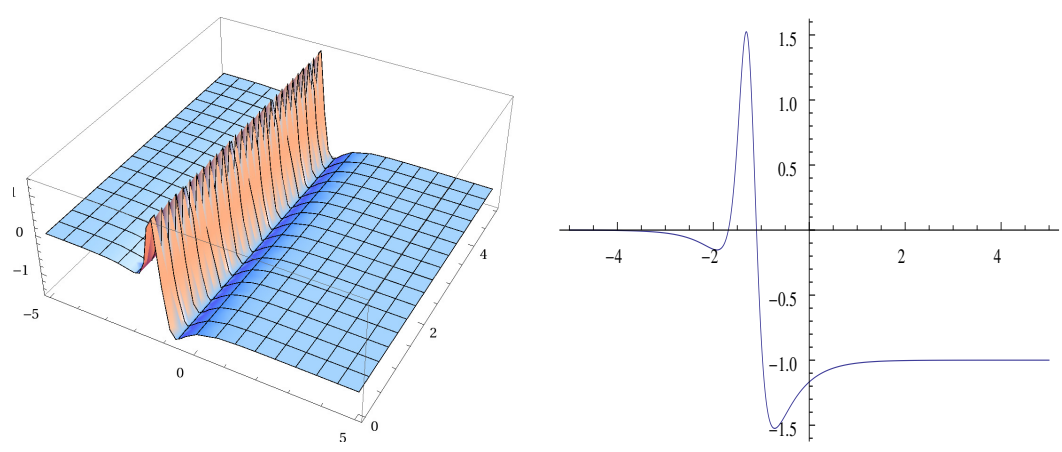

FiguRE 8. $\kappa(y, z)$ of $\xi \backslash\left(\widetilde{\mathrm{PSL}}_{2} \mathbb{R}\right)$ with $\xi(t)=(($ parabolic $), c t)$, $c=9$, and a cross section at $z=0$.

Clearly, $(y, z) \rightarrow(y, z+t)$ is an isometry. From this orthonormal basis $\left\{J\left(X_{1}\right)\right.$, $\left.J\left(X_{2}\right)\right\}$ for the quotient space, or from $\left\{X_{1}, X_{2}\right\}$ together with Formula (0.2), we obtain

$$
\kappa(y, z)=-\frac{c e^{2 y}\left(4 e^{6 y} c^{3}-2 e^{4 y} c^{2}-3 e^{2 y} c+1\right)}{\left(2 e^{4 y} c^{2}-2 e^{2 y} c+1\right)^{2}} .
$$

In particular, if $c=0$, then $\xi(t)$ is a purely parabolic action, and $\kappa(y, z)=0$, and $\xi \backslash \widetilde{P}$ is the flat $\mathbb{R}^{2}$.

6.2.3 $(\xi(t)=(\zeta(t), 2 c t)$, with $(u, v, w)=(0,1,0)$ (with $\zeta(t)$ hyperbolic $))$.

In this case,

$$
\xi(t) \cdot \varphi(x, y, z)=\varphi\left(e^{2 t} x, y+t, z+c t\right)
$$

so that the vertical vector is $V_{(x, y, z)}=(2 x, 1, c)$. The $x z$-plane is a global cross section, and

$$
\begin{aligned}
& X_{1}=\frac{1}{2 \sqrt{(c-x)^{2}+x^{2}}}(2 x-2 c, 0,2 x-c), \\
& X_{2}=\frac{1}{2 \sqrt{\left(c^{2}-2 c x+2 x^{2}\right)\left(c^{2}-2 c x+2 x^{2}+1\right)}}\left(2 x,-c^{2}+2 c x-2 x^{2}, c\right),
\end{aligned}
$$

make an orthonormal basis of the horizontal distribution.

The equation of leaves $\left(e^{2 t} x, y+t, z+c t\right)$ yields the Jacobian of the Riemannian projection on the $x z$-plane

$$
J=\left[\begin{array}{ccc}
1 & -2 x & 0 \\
0 & -c & 1
\end{array}\right]
$$

and, this yields an orthonormal basis for the quotient space

$$
\begin{aligned}
& J\left(X_{1}\right)=\frac{1}{2 \sqrt{(c-x)^{2}+x^{2}}}(2 x-2 c, 2 x-c), \\
& J\left(X_{2}\right)=\frac{\sqrt{c^{2}-2 c x+2 x^{2}+1}}{2 \sqrt{c^{2}-2 c x+2 x^{2}}}(2 x, c) .
\end{aligned}
$$



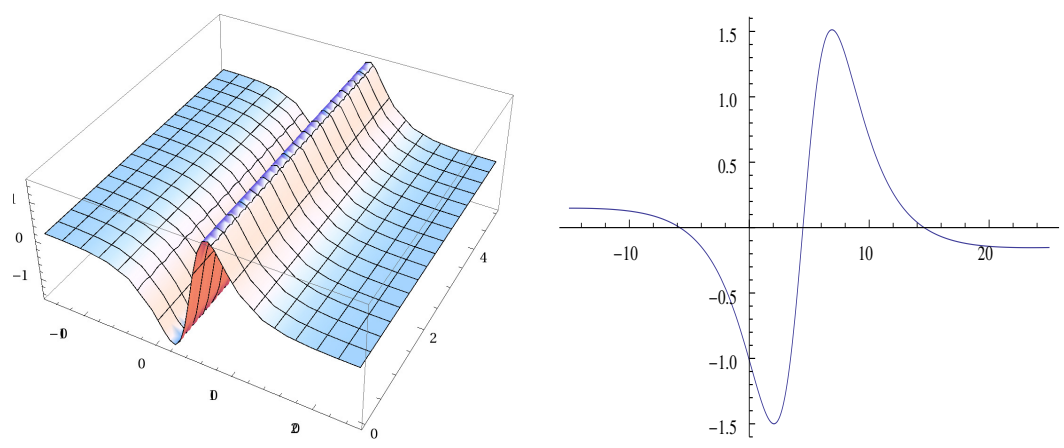

Figure 9. $\kappa(x, z)$ of $\xi \backslash\left(\widetilde{\mathrm{PSL}}_{2} \mathbb{R}\right)$ with $\xi(t)=$ ((hyperbolic), $c t), c=9$, and a cross section at $z=0$.

Clearly, $(y, z) \rightarrow(y, z+t)$ is an isometry. From this orthonormal basis $\left\{J\left(X_{1}\right)\right.$, $\left.J\left(X_{2}\right)\right\}$ for the quotient space, or from $\left\{X_{1}, X_{2}\right\}$ together with Formula (0.2), we obtain

$$
\kappa(x, z)=\frac{-c^{4}-3 c^{2}-2 c x^{3}+\left(c^{2}+9\right) c x+\left(3 c^{2}-4\right) x^{2}+1}{\left(c^{2}-2 c x+2 x^{2}+1\right)^{2}} .
$$

6.2.4 $(\xi(t)=(\zeta(t), 2 c t)$, with $(u, v, w)=(0,0,1)$ (with $\zeta(t)$ elliptic), $c \neq-1)$. The fiber on $\mathbb{R}^{3}$ is

$$
\begin{aligned}
& \xi(t) \cdot \varphi(x, y, z) \\
= & \varphi\left(\frac{2 x \cos (2 t)+\left(x^{2}+e^{4 y}-1\right) \sin (2 t)}{x^{2}+2 \sin (2 t) x+e^{4 y}-\left(x^{2}+e^{4 y}-1\right) \cos (2 t)+1},\right. \\
& \frac{1}{2}\left(2 y-\log \left(x^{2}+2 \sin (2 t) x+e^{4 y}-\left(x^{2}+e^{4 y}-1\right) \cos (2 t)+1\right)+\log (2)\right), \\
& \left.\cos ^{-1}\left(\frac{\sqrt{2}\left(\cos (c t+z)(\cos (t)+x \sin (t))-e^{2 y} \sin (t) \sin (c t+z)\right)}{\sqrt{x^{2}+2 \sin (2 t) x+e^{4 y}-\left(x^{2}+e^{4 y}-1\right) \cos (2 t)+1}}\right)\right) .
\end{aligned}
$$

If $c=-1$, then the action has fixed points. Note that

$$
\xi(t)=(\zeta(t), 2 c t)=\ell(2 c t) \circ r(2 c t)=\ell(2 c t) \circ r(2 c t) \circ r(2(c+1) t),
$$

and the conjugation $\ell(2 c t) \circ r(2 c t)$ acts with fixed points. Each orbit is a circle, except on the core line $\varphi(x, y, z)=\varphi(0,0, z)$ where the orbits are points.

We can take the $\varphi$ (xy-plane) as a smooth cross section for our submersion. The vertical vector is

$$
V_{(x, y, z)}=\left(-1+e^{4 y}-x^{2},-x, c+e^{2 y}\right),
$$

and an orthonormal basis of the horizontal distribution is given by

$$
\begin{aligned}
& X_{1}=\left(X_{11}, X_{12}, X_{13}\right), \\
& X_{2}=\left(X_{21}, X_{22}, X_{23}\right),
\end{aligned}
$$


where

$$
\begin{aligned}
& X_{11}=\frac{e^{2 y}\left(-2 c e^{2 y}-x^{2}-e^{4 y}-1\right)}{\sqrt{2\left(\left(x^{2}+1+c e^{2 y}\right)^{2}+\left(e^{4 y}+c e^{2 y}\right)^{2}\right)}}, \\
& X_{12}=0, \\
& X_{13}=\frac{-c e^{2 y}-x^{2}-1}{\sqrt{2\left(\left(x^{2}+1+c e^{2 y}\right)^{2}+\left(e^{\left.\left.4 y+c e^{2 y}\right)^{2}\right)}\right.\right.}} \\
& X_{21}=\frac{1}{D}\left(2 x e^{2 y}\left(x^{2}+1-e^{4 y}\right)\right), \\
& X_{22}=\frac{1}{D}\left(-e^{-2 y}\left(\left(x^{2}+1+c e^{2 y}\right)^{2}+\left(e^{4 y}+c e^{2 y}\right)^{2}\right)\right), \\
& X_{23}=\frac{1}{D}\left(-2 x e^{2 y}\left(c+e^{2 y}\right)\right)
\end{aligned}
$$

and

$D=2 \sqrt{\left(e^{-4 y}\left(\left(x^{2}+1+c e^{2 y}\right)^{2}+\left(e^{4 y}+c e^{2 y}\right)^{2}\right)+2 x^{2}\right)\left(\left(x^{2}+1+c e^{2 y}\right)^{2}+\left(e^{4 y}+c e^{2 y}\right)^{2}\right)}$.

The equation of leaves yields the Jacobian of the Riemannian projection on the $x y$-plane

$$
J=\left[\begin{array}{ccc}
1 & 0 & \frac{x^{2}-e^{4 y}+1}{\left|c+e^{2 y}\right|} \\
0 & 1 & \frac{x}{\left|c+e^{2 y}\right|}
\end{array}\right]
$$

and, this yields an orthonormal basis for the quotient space

$$
\begin{aligned}
& J\left(X_{1}\right)=\left(J_{11}, J_{12}\right), \\
& J\left(X_{2}\right)=\left(J_{21}, J_{22}\right),
\end{aligned}
$$

where

$$
\begin{aligned}
& J_{11}=-\frac{\sqrt{\left(x^{2}+1+c e^{2 y}\right)^{2}+\left(e^{4 y}+c e^{2 y}\right)^{2}}}{\sqrt{2}\left(c+e^{2 y}\right)}, \\
& J_{12}=-\frac{x\left(c e^{2 y}+x^{2}+1\right)}{\sqrt{2}\left(c+e^{2 y}\right) \sqrt{\left(x^{2}+1+c e^{2 y}\right)^{2}+\left(e^{4 y}+c e^{2 y}\right)^{2}}}, \\
& J_{21}=0, \\
& J_{22}=-\frac{e^{-2 y}\left(2 c^{2} e^{4 y}+2 c e^{2 y}\left(x^{2}+e^{4 y}+1\right)+\left(x^{2}+e^{4 y}\right)^{2}+2 x^{2}+1\right)}{2 \sqrt{\left(e^{-4 y}\left(\left(x^{2}+1+c e^{2 y}\right)^{2}+\left(e^{4 y}+c e^{2 y}\right)^{2}\right)+2 x^{2}\right)\left(\left(x^{2}+1+c e^{2 y}\right)^{2}+\left(e^{4 y}+c e^{2 y}\right)^{2}\right)}} .
\end{aligned}
$$

With $c=0$ (so that $\xi(t)$ is the pure left translation by $K$ ),

$$
\begin{aligned}
& J\left(X_{1}\right)=-\frac{e^{-2 y}}{\sqrt{2} \sqrt{\left(x^{2}+1\right)^{2}+e^{8 y}}}\left(\left(x^{2}+1\right)^{2}+e^{8 y}, x\left(x^{2}+1\right)\right), \\
& J\left(X_{2}\right)=-\left(0, \frac{e^{-2 y}\left(2 x^{2}+\left(x^{2}+e^{4 y}\right)^{2}+1\right)}{2 \sqrt{\left(e^{-4 y}\left(\left(x^{2}+1\right)^{2}+e^{8 y}\right)+2 x^{2}\right)\left(\left(x^{2}+1\right)^{2}+e^{8 y}\right)}}\right) .
\end{aligned}
$$



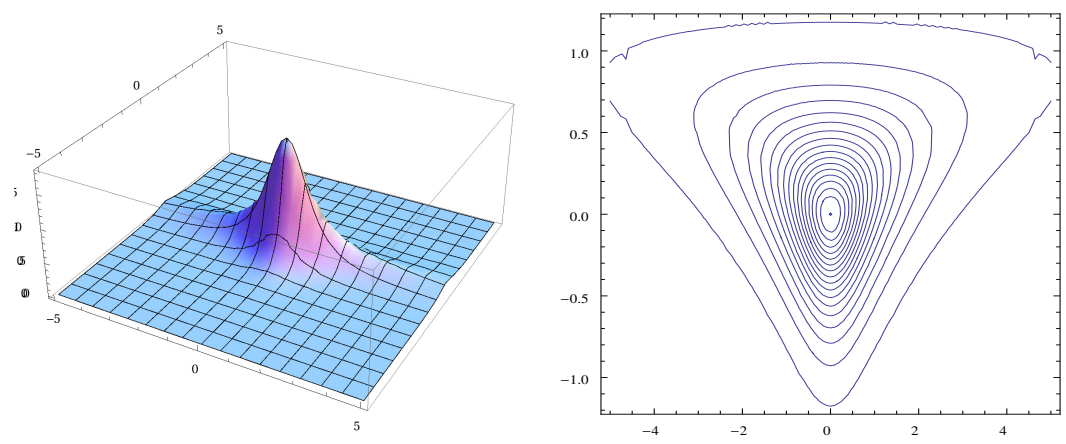

FIGURE 10. $\kappa(x, y)$ of $\xi \backslash\left(\widetilde{\mathrm{PSL}}_{2} \mathbb{R}\right)$ with $\xi(t)=(($ elliptic $), c t)$, $c=0.7$, and the level curves for the curvature.

From the orthonormal basis $\left\{J\left(X_{1}\right), J\left(X_{2}\right)\right\}$ for the quotient space, or from $\left\{X_{1}, X_{2}\right\}$ together with Formula $(0.2)$, we obtain

$$
\begin{aligned}
\kappa(x, y) \cdot D= & c\left(x^{2}+1\right)^{3} e^{2 y}+\left(3 c^{2}+4\right)\left(x^{2}+1\right)^{2} e^{4 y} \\
& -c\left(x^{2}+1\right)\left(2 c^{2}-3\left(x^{2}+7\right)\right) e^{6 y} \\
& -2\left(2 c^{4}-3 c^{2}\left(x^{2}+3\right)-4 x^{2}-6\right) e^{8 y} \\
& -c\left(2 c^{2}-3\left(x^{2}+7\right)\right) e^{10 y}+\left(3 c^{2}+4\right) e^{12 y}+c e^{14 y}
\end{aligned}
$$

where

$$
D=\left(\left(x^{2}+1\right)^{2}+2 c\left(x^{2}+1\right) e^{2 y}+2\left(c^{2}+x^{2}\right) e^{4 y}+2 c e^{6 y}+e^{8 y}\right)^{2} .
$$

In particular, if $c=0$, then $\xi(t)$ is a (left) elliptic action, and

$$
\kappa(x, y)=\frac{4 e^{4 y}\left(\left(x^{2}+1\right)^{2}+e^{8 y}+e^{4 y}\left(2 x^{2}+3\right)\right)}{\left(2 e^{4 y} x^{2}+e^{8 y}+\left(x^{2}+1\right)^{2}\right)^{2}} .
$$

The subgroup $\ell(\widetilde{K}) \times_{\mathbb{Z}} r(\widetilde{K})$ (which is a cylinder) of $\operatorname{Isom}(\widetilde{P})$ commutes with $\xi(t)$, and induces an effective 1 -dimensional isometric action on the base $\xi \backslash \widetilde{P}$. Since

$$
\ell(\widetilde{K}) \backslash \widetilde{P} / r(\widetilde{K})=\ell(\widetilde{K}) \backslash \mathbb{H}^{2} \cong \mathbb{R}^{+}=\{(x, 0): x \geq 0\},
$$

clearly, $\ell(\widetilde{K}) \times_{\mathbb{Z}} r(\widetilde{K}) \cdot \mathbb{R}^{+}=\widetilde{P}$. To measure the behavior of $\kappa$, it is enough to look $\kappa(x, 0)$ for $x \geq 0$.

$$
\kappa(x, 0)=\frac{c x^{6}+(3 c(c+2)+4) x^{4}-2(c-8)(c+1)^{2} x^{2}-4(c+1)^{2}((c-1) c-5)}{\left(x^{4}+2(c+2) x^{2}+2(c+1)^{2}\right)^{2}} .
$$


Theorem 6.3. Let $\xi(t)=(\zeta(t), 2 c t) \in \widetilde{P} \times_{\mathbb{Z}} \mathbb{R}=\operatorname{Isom}_{0}(\widetilde{P})$ be a free, proper action of 1-dimensional subgroup of isometries of $\widetilde{P}$. Let

$$
\mathbb{R} \rightarrow \widetilde{P} \rightarrow \xi \backslash(\widetilde{P})
$$

be the Riemannian submersion given by the action of $\xi$. The curvature $\kappa$ of the quotient space $\xi \backslash \widetilde{P}$ is as follows:

(1) $\xi(t)=(\zeta(t), 2 c t)$, with $(u, v, w)=(0,0,0) \quad($ with $\zeta(t)$ trivial $)$.

The group is the right translation by $\widetilde{K}$, and the orbit space by $\xi$ is the genuine $\mathbb{H}^{2}$ (with $\kappa=-1$ ).

(2) $\xi(t)=(\zeta(t), 2 c t)$, with $(u, v, w)=(1,0,0) \quad$ (with $\zeta(t)$ parabolic).

$$
\kappa(y, z)=-\frac{c e^{2 y}\left(4 e^{6 y} c^{3}-2 e^{4 y} c^{2}-3 e^{2 y} c+1\right)}{\left(2 e^{4 y} c^{2}-2 e^{2 y} c+1\right)^{2}} .
$$

In particular, if $c=0$, then $\xi(t)$ is a purely parabolic action, and $\kappa(y, z)=0$ on $N \backslash \widetilde{P}$ so that $N \backslash \widetilde{P}$ is the flat $\mathbb{R}^{2}$. If $c \neq 0$, then $\operatorname{Isom}_{0}(\xi \backslash \widetilde{P})=\mathbb{R}$.

(3) $\xi(t)=(\zeta(t), 2 c t)$ with $(u, v, w)=(0,1,0) \quad($ with $\zeta(t)$ hyperbolic $)$.

$$
\kappa(x, z)=\frac{-c^{4}-3 c^{2}-2 x^{3} c+\left(c^{2}+9\right) x c+\left(3 c^{2}-4\right) x^{2}+1}{\left(c^{2}-2 x c+2 x^{2}+1\right)^{2}},
$$

and if $c \neq 0$, then $\operatorname{Isom}_{0}(\xi \backslash \widetilde{P})=\mathbb{R}$.

(4) $\xi(t)=(\zeta(t), 2 c t)$, with $(u, v, w)=(0,0,1) \quad$ (with $\zeta(t)$ elliptic $), c \neq$ -1 and If $c \neq 0$, then $\operatorname{Isom}_{0}(\xi \backslash \widetilde{P})=\mathrm{SO}(2)$.

$$
\begin{aligned}
\kappa(x, y) \cdot D= & c\left(x^{2}+1\right)^{3} e^{2 y}+\left(3 c^{2}+4\right)\left(x^{2}+1\right)^{2} e^{4 y} \\
& -c\left(x^{2}+1\right)\left(2 c^{2}-3\left(x^{2}+7\right)\right) e^{6 y} \\
& -2\left(2 c^{4}-3 c^{2}\left(x^{2}+3\right)-4 x^{2}-6\right) e^{8 y} \\
& -c\left(2 c^{2}-3\left(x^{2}+7\right)\right) e^{10 y}+\left(3 c^{2}+4\right) e^{12 y}+c e^{14 y},
\end{aligned}
$$

where

$$
D=\left(\left(x^{2}+1\right)^{2}+2 c\left(x^{2}+1\right) e^{2 y}+2\left(c^{2}+x^{2}\right) e^{4 y}+2 c e^{6 y}+e^{8 y}\right)^{2} .
$$

In particular, if $c=0$, then $\xi(t)$ is a (left) elliptic action, and

$$
\kappa(x, y)=\frac{4 e^{4 y}\left(\left(x^{2}+1\right)^{2}+e^{8 y}+e^{4 y}\left(2 x^{2}+3\right)\right)}{\left(2 e^{4 y} x^{2}+e^{8 y}+\left(x^{2}+1\right)^{2}\right)^{2}} .
$$

The isometry group is $\operatorname{Isom}_{0}(\xi \backslash \widetilde{P})=\mathrm{SO}(2)$.

Remark 6.4. In the elliptic case (4), for $c=-1, \xi(t)$ does not define a foliation (since the action is not free). $\lim _{c \rightarrow \infty} \kappa(x, 0)=-1$ and the space gets closer to $\mathbb{H}^{2}$, because the action gets closer to the right $K$-action. For any $c, \lim _{x \rightarrow \infty} \kappa(x, 0)=0$. The normalizer of $\xi$ is $\widetilde{\mathrm{SO}}(2) \times_{\mathbb{Z}} \widetilde{\mathrm{SO}}(2)$, a cylinder, 
generated by the left and right $\widetilde{K}$-actions. Thus, $\operatorname{Isom}_{0}(\xi \backslash \widetilde{P})=N_{0} / G=$ $\widetilde{\mathrm{SO}}(2) \times_{\mathbb{Z}} \widetilde{\mathrm{SO}}(2) / \widetilde{\mathrm{SO}}(2) \cong \mathbb{R} \times \mathrm{SO}(2) / \mathbb{R} \cong \mathrm{SO}(2)$.

\section{The solvable Lie group Sol}

7.1. We express the group Sol as a matrix subgroup

$$
\mathrm{Sol}=\left\{\left[\begin{array}{ccc}
e^{z} & 0 & x \\
0 & e^{-z} & y \\
0 & 0 & 1
\end{array}\right] \mid x, y, z \in \mathbb{R}\right\} .
$$

The standard left-invariant Riemannian metric is given by the orthonormal basis

$$
E_{1}=\left[\begin{array}{lll}
0 & 0 & 1 \\
0 & 0 & 0 \\
0 & 0 & 0
\end{array}\right], E_{2}=\left[\begin{array}{lll}
0 & 0 & 0 \\
0 & 0 & 1 \\
0 & 0 & 0
\end{array}\right], E_{3}=\left[\begin{array}{ccc}
1 & 0 & 0 \\
0 & -1 & 0 \\
0 & 0 & 0
\end{array}\right] .
$$

Then the group of isometries is $\operatorname{Isom}_{0}(\mathrm{Sol})=$ Sol. Define the global coordinate system on Sol by

$$
\varphi(x, y, z)=e^{x E_{1}} e^{y E_{2}} e^{z E_{3}}=\left[\begin{array}{ccc}
e^{z} & 0 & x \\
0 & e^{-z} & y \\
0 & 0 & 1
\end{array}\right] .
$$

7.2. Let $\xi(t)$ be the 1-parameter group of isometries generated by an Lie algebra element $u E_{1}+v E_{2}+w E_{3}$.

If $w \neq 0$, conjugation by $\varphi\left(\frac{u}{w}, \frac{v}{w}, 0\right)$ maps $u E_{1}+v E_{2}+w E_{3}$ to $w E_{3}$. By reparametrizing, we may assume $w=1$. Thus,

$$
\xi(t) \cdot \varphi(x, y, z)=\left\{\begin{array}{lll}
\varphi\left(x e^{t}, y e^{-t}, z+t\right), & \text { if } & (u, v, w)=(0,0,1), \\
\varphi(u t+x, v t+y, z), & \text { if } \quad(u, v, w)=(u, v, 0) .
\end{array}\right.
$$

The vertical vector on $\mathbb{R}^{3}$ is

$$
V_{(x, y, z)}=e^{-z}(u+w x) \frac{\partial}{\partial x}+e^{z}(v-w y) \frac{\partial}{\partial y}+w \frac{\partial}{\partial z} .
$$

7.2.1 $((u, v, w)=(0,0,1))$.

In this case,

$$
\xi(t) \cdot \varphi(x, y, z)=\varphi\left(x e^{t}, y e^{-t}, z+t\right)
$$

so that the vertical vector is $V_{(x, y, z)}=(x,-y, 1)$. The $x y$-plane is a global cross section, and

$$
\begin{aligned}
& X_{1}=\frac{1}{\sqrt{x^{2}+1}}(-1,0, x), \\
& X_{2}=\frac{-1}{\sqrt{\left(x^{2}+1\right)\left(x^{2}+y^{2}+1\right)}}\left(x y, x^{2}+1, y\right),
\end{aligned}
$$

make an orthonormal basis of the horizontal distribution.

The equation of leaves $\left(x e^{t}, y e^{-t}, z+t\right)$ yields the Jacobian of the Riemannian projection on $x y$-plane

$$
J=\left[\begin{array}{rrr}
1 & 0 & -x \\
0 & 1 & y
\end{array}\right]
$$



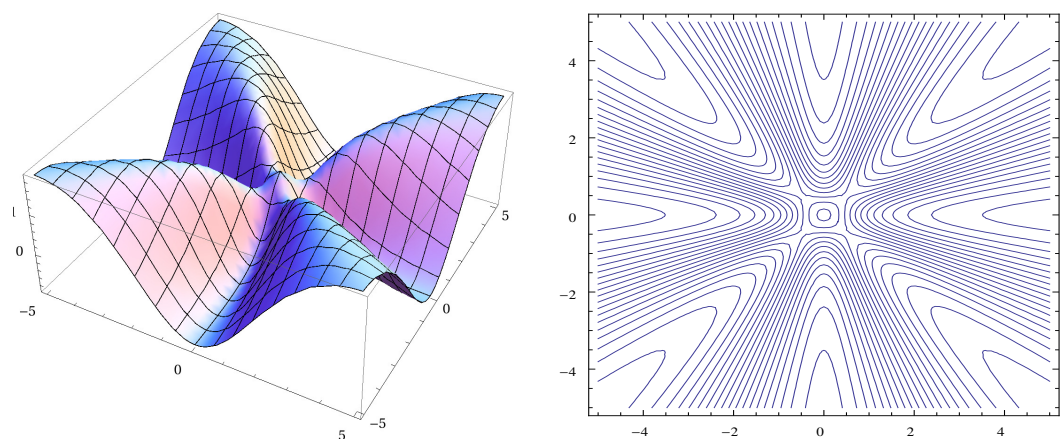

Figure 11. Curvature of $\xi \backslash(\mathrm{Sol})$ with $\xi(t)=(0,0, t)$ and the level curves for the curvature (No group actions).

and, this yields an orthonormal basis for the quotient space

$$
\begin{aligned}
& J\left(X_{1}\right)=\frac{1}{\sqrt{x^{2}+1}}\left(-\left(x^{2}+1\right), x y\right), \\
& J\left(X_{2}\right)=\frac{1}{\sqrt{x^{2}+1}}\left(0,-\sqrt{x^{2}+y^{2}+1}\right) .
\end{aligned}
$$

From this orthonormal basis $\left\{J\left(X_{1}\right), J\left(X_{2}\right)\right\}$ for the quotient space, or from $\left\{X_{1}, X_{2}\right\}$ together with Formula (0.2), we obtain [3],

$$
\kappa(x, y)=-\frac{-1+x^{4}-10 x^{2} y^{2}+y^{4}}{\left(1+x^{2}+y^{2}\right)^{2}} .
$$

7.2.2 $((u, v, w)=(u, v, 0))$.

In this case,

$$
\xi(t) \cdot \varphi(x, y, z)=\varphi(u t+x, v t+y, z)
$$

so that the vertical vector is $V_{(x, y, z)}=(u, v, 0)$. Since one of $u$ and $v$ is non-zero, we assume $u \neq 0$. Then the $y z$-plane is a global cross section, and

$$
\begin{aligned}
& X_{1}=(0,0,1), \\
& X_{2}=\frac{1}{\sqrt{u^{2}+e^{4 z} v^{2}}}\left(e^{3 z} v,-e^{-z} u\right),
\end{aligned}
$$

make an orthonormal basis of the horizontal distribution.

The equation of leaves $(u t+x, v t+y, z)$ yields the Jacobian of the Riemannian projection on the $x z$-plane

$$
J=\left[\begin{array}{ccc}
-\frac{v}{u} & 1 & 0 \\
0 & 0 & 1
\end{array}\right]
$$

and, this yields an orthonormal basis for the quotient space

$$
J\left(X_{1}\right)=(0,1),
$$




$$
J\left(X_{2}\right)=\left(-\frac{\sqrt{u^{2}+e^{4 z} v^{2}}}{u e^{z}}, 0\right) .
$$

One can easily see that $\operatorname{Isom}_{0}(\xi \backslash \mathrm{Sol})=\mathbb{R}$. From this orthonormal basis $\left\{J\left(X_{1}\right), J\left(X_{2}\right)\right\}$ for the quotient space, or from $\left\{X_{1}, X_{2}\right\}$ together with Formula (0.2), we obtain

$$
\kappa(y, z)=\frac{12 u^{2} v^{2} e^{4 z}}{\left(u^{2}+v^{2} e^{4 z}\right)^{2}}-1 .
$$

Theorem 7.3. A 1-dimensional closed subgroup $G$ of Sol is either $\xi(t)=e^{t E_{3}}$ or $\xi(t)=e^{t\left(a E_{1}+b E_{2}\right)}$, up to conjugation. Let

$$
\mathbb{R} \longrightarrow \text { Sol } \longrightarrow \xi \backslash \text { Sol }
$$

be the Riemannian submersion given by the action of $\xi$.

(1) $\xi(t)=e^{t E_{3}}:$

$$
\kappa(x, y)=-\frac{-1+x^{4}-10 x^{2} y^{2}+y^{4}}{\left(1+x^{2}+y^{2}\right)^{2}} .
$$

This is the only case where the quotient space has 0-dimensional isometry group.

(2) $\xi(t)=e^{t\left(a E_{1}+b E_{2}\right)}$ :

$$
\kappa(y, z)=-1+\frac{12 a^{2} b^{2} e^{4 z}}{\left(a^{2}+b^{2} e^{4 z}\right)^{2}}, \text { and } \operatorname{Isom}_{0}(\xi \backslash \operatorname{Sol})=\mathbb{R} .
$$

Proof. (1) To see that $\operatorname{Isom}_{0}(\xi \backslash$ Sol $)$ is trivial, we calculate the critical points of the curvature to find $(x, y)=\left( \pm \frac{1}{2}, \pm \frac{1}{2}\right)$ or $(0,0)$ with $\kappa(0,0)=1$ (local maximum) and $\kappa\left( \pm \frac{1}{2}, \pm \frac{1}{2}\right)=\frac{2}{3}$ (four saddle points). Suppose $\operatorname{Isom}_{0}(\xi \backslash$ Sol) is nontrivial. Then the group must fix these isolated points. This implies the group fixes the four level curves $\kappa=\frac{2}{3}$ connecting the saddle points. The 1-dimensional isometric action will be a "circular rotation" around the origin, near the origin. Such an action becomes discontinuous at the saddle points. We conclude that $\operatorname{Isom}_{0}(\xi \backslash$ Sol $)$ is trivial.

(2) The normalizer $N_{0}$ of $\xi(t)$ in $\operatorname{Isom}_{0}(\mathrm{Sol})=$ Sol is $\mathbb{R}^{2}$ containing $e^{E_{1}}$ and $e^{E_{2}}$, and $\operatorname{Isom}_{0}(\xi \backslash \mathrm{Sol})=N_{0} / \xi=\mathbb{R}$.

Remark 7.4. The curvatures are bounded: (1) $-1<\kappa \leq 2$. (2) $2 / 3 \leq \kappa \leq 1$. The case where $\xi(t)=e^{t E_{3}}$ is the only time among all 3 -dimensional model spaces where the orbit space $\xi \backslash X$ has 0 -dimensional isometry group.

\section{The hyperbolic space $\mathbb{H}^{3}$}

8.1. The isometry group of $\mathbb{H}^{3}$ is $\operatorname{PSL}(2, \mathbb{C})$. The action is given as follows: The linear fractional transformation on quaternions, mapping $w \rightarrow(a w+b)(c w+$ $d)^{-1}$ leaves the subset

$$
\mathbb{H}^{3}=\{x+y i+z j+0 k: z>0\}
$$



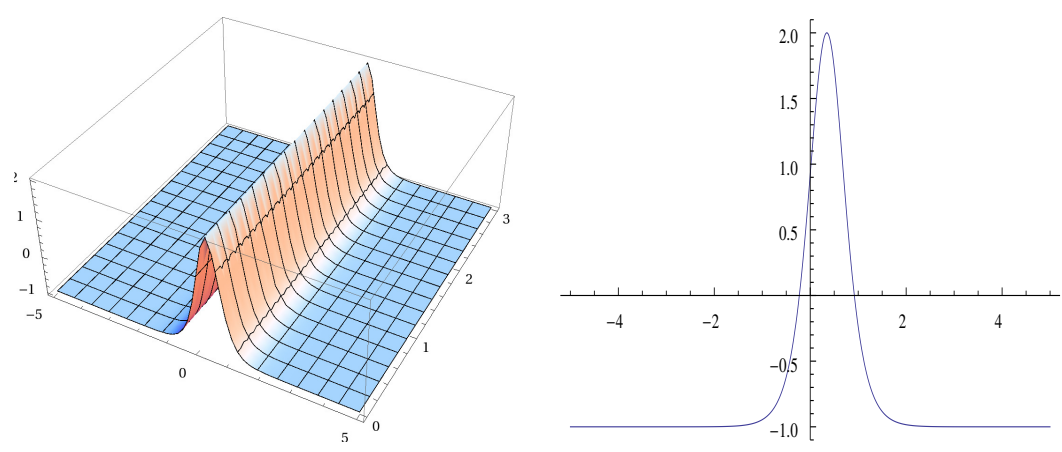

Figure 12. Curvature $\kappa(y, z)$ of $\xi \backslash(\mathrm{Sol})$ with $\xi(t)=(2 t, t, 0)$ and a cross section $y=0$.

invariant. It turns out that

$$
\left[\begin{array}{ll}
a & b \\
c & d
\end{array}\right]: x+y i+z j \mapsto(a(x+y i+z j)+b)(c(x+y i+z j)+d)^{-1}
$$

is an isometric action of $\operatorname{PSL}(2, \mathbb{C})$ on $\mathbb{H}^{3}$. For our calculation, we need the following result [4]: The matrix

$$
\left[\begin{array}{ll}
a_{11}+b_{11} i & a_{12}+b_{12} i \\
a_{21}+b_{21} i & a_{22}+b_{22} i
\end{array}\right] \in \mathrm{SL}(2, \mathbb{C})
$$

$\operatorname{maps}(x, y, z, 0)$ to

$\left(\frac{2 a_{11} a_{22} x-x+b_{12}\left(b_{22}+2 b_{21} x\right)+2 a_{11} b_{22} y+a_{12}\left(a_{22}-2 b_{21} y\right)+a_{11} a_{21} r^{2}+b_{11} b_{21} r^{2}}{a_{22}{ }^{2}+2\left(a_{21} x-b_{21} y\right) a_{22}+b_{22}{ }^{2}+2 b_{22}\left(b_{21} x+a_{21} y\right)+\left(a_{21}{ }^{2}+b_{21}{ }^{2}\right) r^{2}}\right.$, $\frac{a_{22} b_{12}+2 a_{21} x b_{12}-2 b_{21} y b_{12}-a_{12} b_{22}-2 a_{11} b_{22} x+2 b_{11} b_{22} y+y+a_{21} b_{11} r^{2}-a_{11} b_{21} r^{2}}{a_{22}{ }^{2}+2\left(a_{21} x-b_{21} y\right) a_{22}+b_{22}{ }^{2}+2 b_{22}\left(b_{21} x+a_{21} y\right)+\left(a_{21}{ }^{2}+b_{21}{ }^{2}\right) r^{2}}$, $\left.\frac{z}{a_{22}^{2}+2\left(a_{21} x-b_{21} y\right) a_{22}+b_{22}^{2}+2 b_{22} b_{21} r^{2}}, 0\right)$,

where $r^{2}=x^{2}+y^{2}+z^{2}$.

8.2. A 1-dimensional subgroup of $\operatorname{SL}(2, \mathbb{C})$ is either elliptic, parabolic or loxodromic, up to conjugation.

8.2.1 (Elliptic: $\left.\xi(t)=\exp \left(t\left[\begin{array}{cc}i & 0 \\ 0 & -i\end{array}\right]\right)\right)$.

Since

$$
\xi(t)(x, y, z, 0)=(x \cos (t)-y \sin (t), y \cos (t)+x \sin (t), z, 0),
$$

elliptic subgroup cannot act freely, so there is no elliptic foliation.

8.2.2 (Parabolic: $\left.\xi(t)=\exp \left(t\left[\begin{array}{cc}0 & c+d i \\ 0 & 0\end{array}\right]\right)\right)$.

Since

$$
\xi(t)(x, y, z, 0)=(c t+x, d t+y, z, 0),
$$


the orbit space $\xi \backslash \mathbb{H}^{3}$ is isometric to the planes parallel to the $z$-axis, and $\kappa=-1$ (Note this is integrable).

8.2.3 (Loxodromic: $\left.\xi(t)=\exp \left(t\left[\begin{array}{cc}a+b i & 0 \\ 0 & -a-b i\end{array}\right]\right), a \neq 0\right)$.

$$
\xi(t)(x, y, z, 0)=e^{2 a t}(x \cos (2 b t)-y \sin (2 b t), y \cos (2 b t)+x \sin (2 b t), z, 0) .
$$

If $b=0$, it is hyperbolic. Otherwise, it is expanding while rotating. Easily, the plane $z=1$ is a global cross section. The vertical vector field is given by

$$
V=(2 a x-2 b y, 2 b x+2 a y, 2 a)
$$

and the horizontal distribution is given by an orthogonal basis

$$
\begin{aligned}
& X_{1}=(-a, 0, a x-b y), \\
& X_{2}=\left(a b x^{2}+a^{2} x y-b^{2} x y-a b y^{2},-a^{2} x^{2}+8 a b x y-b^{2} y^{2}-a^{2}, a b x z+a^{2} y\right) .
\end{aligned}
$$

The equation of leaves yields the Jacobian of the Riemannian projection on the $x y$-plane $(z=1)$

$$
J=\left[\begin{array}{lll}
1 & 0 & \frac{1}{a}(b y-a x) \\
0 & 1 & \frac{1}{a}(b x-a y)
\end{array}\right]
$$

and, this yields an orthogonal basis for the quotient space

$$
\begin{aligned}
& J\left(X_{1}\right)=\left(-a-\frac{1}{a}(b y-a x)^{2}, \frac{1}{a}(b x-a y)(a x-b y)\right), \\
& J\left(X_{2}\right)=\left(0,\left(b^{2}-a^{2}\right) x^{2}+2 a b x y-\left(a^{2}+b^{2}\right) y^{2}-a^{2}\right) .
\end{aligned}
$$

Using Formula (0.2), we obtain

$$
\kappa(x, y)=-1+\left(3 a^{2} b^{2}\right) /\left(b^{2}\left(x^{2}+y^{2}\right)+a^{2}\left(1+x^{2}+y^{2}\right)\right)^{2} .
$$

Theorem 8.3. Let $\xi(t) \in S L(2, \mathbb{C})=\operatorname{Isom}_{0}\left(\mathbb{H}^{3}\right)$ be a free, proper action of 1-dimensional subgroup of isometries of $\mathbb{H}^{3}$. Let

$$
\mathbb{R} \rightarrow \mathbb{H}^{3} \rightarrow \xi \backslash \mathbb{H}^{3}
$$

be the Riemannian submersion given by the action of $\xi$. The curvature $\kappa$ of the quotient space $\xi \backslash \mathbb{H}^{3}$ is as follows:

(1) Elliptic. $\xi(t)=\exp \left(t\left[\begin{array}{cc}i & 0 \\ 0 & -i\end{array}\right]\right)$.

There is no elliptic foliation.

(2) Parabolic. $\xi(t)=\exp \left(t\left[\begin{array}{cc}0 & c+d i \\ 0 & 0\end{array}\right]\right)$.

The orbit space $\xi \backslash \mathbb{H}^{3}$ is isometric to the plane parallel to the $z$-axis, and $\kappa=-1$ (Note this is integrable).

(3) Loxodromic. $\xi(t)=\exp \left(t\left[\begin{array}{cc}a+b i & 0 \\ 0 & -a-b i\end{array}\right]\right), a \neq 0$.

$$
\kappa(x, y)=-1+\frac{3 a^{2} b^{2}}{\left(b^{2}\left(x^{2}+y^{2}\right)+a^{2}\left(1+x^{2}+y^{2}\right)\right)^{2}} \text {, and } \operatorname{Isom}_{0}\left(\xi \backslash \mathbb{H}^{3}\right)=\mathrm{SO}(2) .
$$

Proof. (3) The normalizer of $\xi(t)$ consists of $\left[\begin{array}{cc}z & 0 \\ 0 & z^{-1}\end{array}\right]$ so that $N_{0}=\mathbb{C}^{*}$. Therefore, $\operatorname{Isom}_{0}\left(\xi \backslash \mathbb{H}^{3}\right)=\mathbb{C}^{*} / \mathbb{R}=\mathrm{SO}(2)$. 

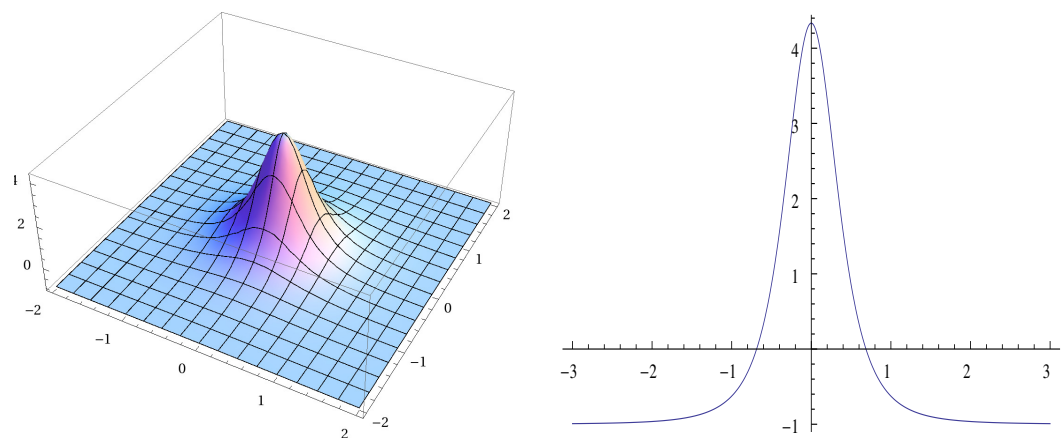

Figure 13. Curvature $\kappa(y, z)$ of $\xi \backslash(\mathrm{Sol})$ with $\xi(t)$ loxodromic with $a=3, b=4$ and a cross section $y=0$.

Remark 8.4. (3) On the $x y$-plane, with the polar coordinate,

$$
\kappa(r, \theta)=\kappa(r)=-1+\frac{3 a^{2} b^{2}}{\left(b^{2} r^{2}+a^{2}\left(1+r^{2}\right)\right)^{2}} .
$$

If $b=0$, the action is purely hyperbolic, and $\kappa=-1$. Otherwise, maximum $\kappa=-1+\frac{3 b^{2}}{a^{2}}$ occurs at $(0,0)$, and $-1<\kappa \leq-1+\frac{3 b^{2}}{a^{2}}$.

\section{References}

[1] D. Gromoll and K. Grove, One-dimensional metric foliations in constant curvature spaces, Differential geometry and complex analysis, 165-168, Springer, Berlin, 1985.

[2] S. Helgason, Differential Geometry, Lie groups, and Symmetric Spaces, Academic Press, 1978.

[3] B. O'Neill, The fundamental equations of a submersion, Michigan Math. J. 13 (1966), 445-457.

[4] J. Ratcliffe, Foundations of Hyperbolic Manifolds, Springer, 2006.

[5] P. Scott, The geometries of 3-manifolds, Bull. London Math. Soc. 15 (1983), no. 5, 401-487.

Kyung Bai Lee

Department of Mathematics

UNIVERSITY OF OKLAHOMA

NORMAN, OK 73019, USA

E-mail address: kblee@math.ou.edu

JoOnSANG PARK

Department of Mathematics

DONGGUK UNIVERSITY

SeOul 100-715, Korea

E-mail address: jpark@dgu.edu 